ARTICLE

\title{
Suppression of mitochondrial ROS by prohibitin drives glioblastoma progression and therapeutic resistance
}

Haohao Huang1,2,3,7, Songyang Zhang ${ }^{1,7}$, Yuanyuan Li ${ }^{1,7}$, Zhaodan Liu', Lanjuan Mi', Yan Cai ${ }^{1}$, Xinzheng Wang ${ }^{1}$, Lishu Chen ${ }^{1}$, Haowen Ran ${ }^{1}$, Dake Xiao ${ }^{1}$, Fangye Li ${ }^{4}$, Jiaqi Wu1, Tingting Li ${ }^{1}$, Qiuying Han ${ }^{1}$, Liang Chen ${ }^{1}$, Xin Pan (1) ${ }^{1}$,

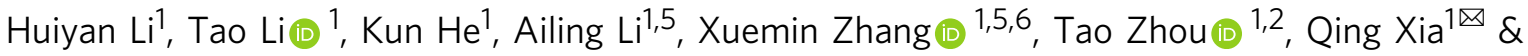
Jianghong Man (1) 1,2凶

Low levels of reactive oxygen species (ROS) are crucial for maintaining cancer stem cells (CSCs) and their ability to resist therapy, but the ROS regulatory mechanisms in CSCs remains to be explored. Here, we discover that prohibitin (PHB) specifically regulates mitochondrial ROS production in glioma stem-like cells (GSCs) and facilitates GSC radiotherapeutic resistance. We find that PHB is upregulated in GSCs and is associated with malignant gliomas progression and poor prognosis. PHB binds to peroxiredoxin3 (PRDX3), a mitochondrion-specific peroxidase, and stabilizes PRDX3 protein through the ubiquitinproteasome pathway. Knockout of PHB dramatically elevates ROS levels, thereby inhibiting GSC self-renewal. Importantly, deletion or pharmacological inhibition of PHB potently slows tumor growth and sensitizes tumors to radiotherapy, thus providing significant survival benefits in GSC-derived orthotopic tumors and glioblastoma patient-derived xenografts. These results reveal a selective role of PHB in mitochondrial ROS regulation in GSCs and suggest that targeting PHB improves radiotherapeutic efficacy in glioblastoma.

\footnotetext{
${ }^{1}$ State Key Laboratory of Proteomics, National Center of Biomedical Analysis, Beijing, China. ${ }^{2}$ Nanhu Laboratory, Jiaxing, Zhejiang Province, China. ${ }^{3}$ Department of Neurosurgery, General Hospital of Central Theater Command of Chinese People's Liberation Army, Wuhan, PR China. ${ }^{4}$ Department of Neurosurgery, First Medical Center of PLA General Hospital, Beijing, China. ${ }^{5}$ The First Hospital of Jilin University, Changchun, China. ${ }^{6}$ State Key Laboratory of Toxicology and Medical Countermeasures, Beijing Institute of Pharmacology and Toxicology, National Center of Biomedical Analysis, Beijing, China. ${ }^{7}$ These

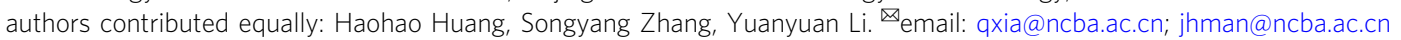


G lioblastoma (GBM) is the most common and lethal type of human primary brain tumor with an extremely poor prognosis ${ }^{1-3}$. Ionizing radiation (IR) is one of the standard nonsurgical treatments for nearly all $\mathrm{GBM}^{4,5}$. Unfortunately, the efficacy of radiotherapy in GBM is dismal partially due to the radioresistance of cancer stem cells (CSCs) in GBM, termed glioma stem-like cells $(\mathrm{GSCs})^{6-10}$. CSCs are functionally defined by their capacity to maintain tumor heterogeneity, drive tumor growth, and therapy resistance ${ }^{9,11}$. Currently, there is no effective targeted therapy for CSCs in GBM treatment. Thus, the identification of essential GSC-specific regulators may provide insights into novel therapeutic strategies against GSCs.

Reactive oxygen species (ROS) are important byproducts of cell metabolism. The maintenance of ROS homeostasis is crucial for cancer cell survival ${ }^{12-14}$. Similar to normal tissue stem cells, CSCs may contain lower levels of ROS relative to bulk tumor cells (BTCs), and this promotes CSC self-renewal and tumor therapeutic resistance ${ }^{15}$. This raises a critical question of whether CSCs and normal stem cells share the same mechanisms to meet redox stress, a process that poses a major challenge for targeting ROS as a radical therapeutic approach for CSCs. However, the regulatory mechanisms of ROS in CSCs are not fully understood.

To adapt to oxidative stress, cancer cells develop a ROSscavenging system to eliminate the over-accumulation of $\mathrm{ROS}^{16-19}$. Peroxiredoxins (PRDXs) are a family of thiol peroxidases that scavenge peroxides in cells. Mammalian cells possess six PRDX isoforms, with PRDX1, 2, and 6 in the cytoplasm, PRDX3 in the mitochondria, PRDX4 in the endoplasmic reticulum, and PRDX5 distributed throughout of the cell ${ }^{20}$. The PRDX family proteins are divided into three classes (2-Cys, atypical 2Cys, and 1-Cys) based on the number of Cys residues that participate in catalysis. PRDX3 belongs to the 2-Cys subgroup and exists as a homodimer. In the catalytic cycle of PRDXs, $\mathrm{H}_{2} \mathrm{O}_{2}$ oxidizes the catalytic thiol (Cys-SH) to sulfenic acid (Cys-SOH), and this then reacts with the second conserved Cys-SH of the other subunit in the homodimer to form an intersubunit disulfide. This disulfide is subsequently reduced by thioredoxin (Trx) to complete the catalytic cycle ${ }^{21,22}$. Mitochondria are a major source of ROS. Kinetic analysis indicates that PRDX3 contributes to the majority of hydrogen peroxide reduction in mitochondria ${ }^{19,22}$, suggesting a crucial role for PRDX3 in the maintenance of mitochondrial redox balance. Although members of the PRDX family have been linked to malignant progression and chemotherapeutic resistance in certain types of tumors ${ }^{20,23}$, the role of PRDX3 in CSCs remains unknown.

Prohibitin (PHB) and its homolog PHB2, comprising the $\mathrm{PHB}$ family of proteins, are highly conserved pleiotropic proteins with multiple functions in different cell types ${ }^{24-26}$. PHB was originally identified as a tumor suppressor, as it co-localizes with p53 and $\mathrm{pRb}$ in the nucleus of breast cancer cells and promotes cell apoptosis ${ }^{27,28}$. It has also been suggested that PHB is localized in the plasma membrane where it activates PI3K/Akt and C-Raf/ ERK signaling pathways in cervical carcinoma cell lines and $\mathrm{T}$ cells ${ }^{29,30}$. Recently, a study has shown that PHB forms a complex with HIRA in the nucleus of human embryonic stem cells to regulate chromatin organization, suggesting an unexpected role of PHB in stem cell maintenance ${ }^{31}$. While the function of PHB is controversial, most studies have provided insights into the roles of PHB in mitochondrial biology ${ }^{25}$. The diverse functions of PHB may be modulated in a tissue-dependent manner, thus indicating that targeting PHB could provide a useful strategy for cancer treatment. In this study, we identified PHB as a specific regulator of ROS in GSCs. PHB is highly expressed in GSCs relative to non-stem tumor cells (NSTCs), neural progenitor cells (NPCs), and normal brain cells. PHB binds to and stabilizes PRDX3, to thereby inhibit mitochondrial
ROS accumulation and promote GSC self-renewal and radioresistance. Targeting GSCs by genetic deletion or pharmacological inhibition of PHB potently inhibits GBM growth and overcomes the resistance of GBM to radiation treatment, highlighting that PHB blockade synergizes with radiotherapy in GBM treatment.

\section{Results}

PHB is highly expressed in GSCs. Our previous mass spectrometry screening identified a series of differentially expressed proteins between GSCs and their matched differentiated $\mathrm{NSTCs}^{32}$. Among them, 35 proteins were upregulated in four GSC lines relative to NSTCs (average fold change >2) (Supplementary Fig. 1a). To select specific candidates for further study, we used the following criteria: (1) the candidate should be abundantly expressed in GSCs; (2) the candidate should be associated with malignant progression of glioma; and (3) the candidate could be targeted by small molecular inhibitors. After candidate filtering with these criteria, we focused on PHB, an evolutionarily conserved and multifunctional protein ${ }^{24,25}$, whose role in GSCs remains unknown.

To confirm the upregulation of PHB in GSCs, we first performed immunoblot (IB) analysis in multiple GSCs that were functionally characterized in studies including ours ${ }^{32-35}$. The results showed that PHB was highly expressed in all six GSC lines relative to the matched NSTCs (Fig. 1a and Supplementary Fig. 1b). PHB2, a homolog of PHB, was only increased in three of the six GSC lines compared with NSTCs (Fig. 1a, 3691, 3832, and H2S). Moreover, PHB levels were gradually decreased during GSC differentiation induced by serum (Supplementary Fig. 1c). Additionally, PHB expression was dramatically elevated in GSCs and tumor cells isolated from GBM specimens, compared to levels in normal human astrocytes (NHA), NPCs, and established glioma cell lines (Fig. 1b, c). To assess the expression of PHB in GSCs in vivo, we performed co-immunofluorescence (Co-IF) staining of PHB and the stem cell markers SOX2 or Olig2 in primary GBM specimens. The results showed that SOX2+ or Olig2 + cells were enriched in cells with high PHB staining (Fig. 1d and Supplementary Fig. 1d). PHB levels were positively correlated with the expression of SOX2 and Olig2 in GBM tissues (Fig. 1e and Supplementary Fig. 1e). We further analyzed two independent Gene Expression Omnibus (GEO) databases (GSE86237 and GSE54791) (36,37 $^{3}$ and we found that $P H B$ was significantly increased in GSCs relative to BTCs (Fig. 1f) or the matched differentiated glioma cells (DGCs) (Supplementary Fig. 1f), supporting the upregulation of PHB in GSCs.

To investigate the clinical relevance of PHB in glioma, we performed IB analysis using fresh GBM specimens. We found that the expression of PHB was strongly increased in GBM tissues relative to adjacent normal brain tissues (Fig. 1g). Immunohistochemical (IHC) staining showed that PHB was strongly expressed in a subpopulation of tumor cells in GBM tissues but was barely expressed in normal brain (Fig. $1 \mathrm{~h}$ and Supplementary Fig. $1 \mathrm{~g}$ ). Moreover, IHC staining using a glioma tissue microarray demonstrated that both the percentage of $\mathrm{PHB}+$ cells and the intensity of PHB staining were significantly higher in high-grade gliomas relative to low-grade gliomas (Fig. 1i and Supplementary Table 1). Importantly, glioma patients with high PHB IHC scores exhibited increased recurrence (Fig. 1j and Supplementary Fig. 1h). In high-grade gliomas (grades III and IV), patients with high PHB IHC scores were associated with poor survival (Fig. 1k).

PHB possesses a highly conserved $3^{\prime}$-untranslated region (UTR) containing a putative microRNA-27a biding site 38 (Supplementary Fig. 2a), suggesting that miR-27a may regulate the expression of PHB in GSCs. To test this possibility, we first 
a
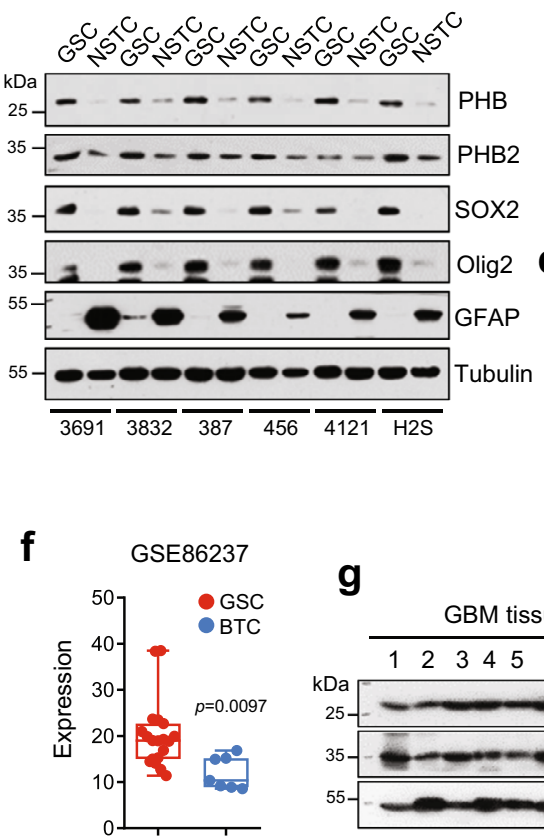

\section{i}

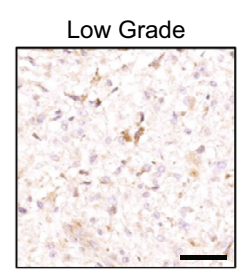

g

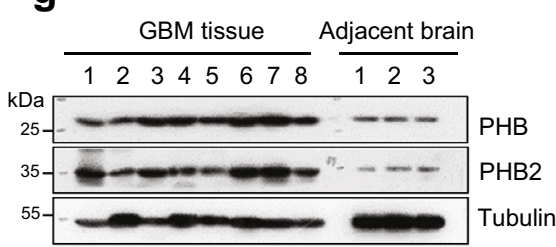

b

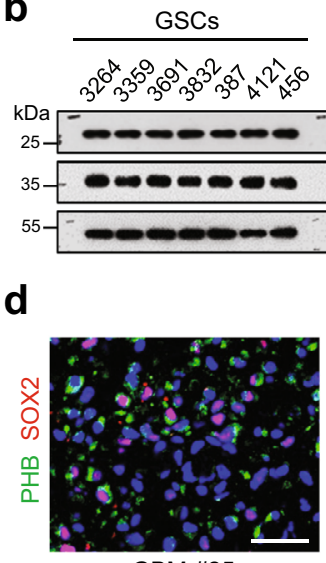

GBM \#25
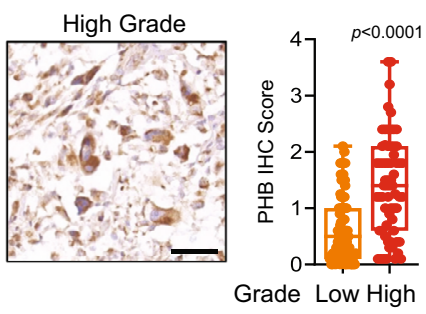

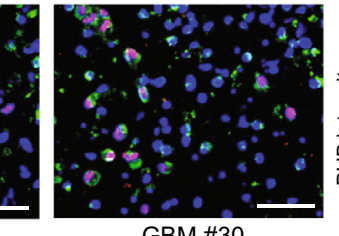

GBM \#30

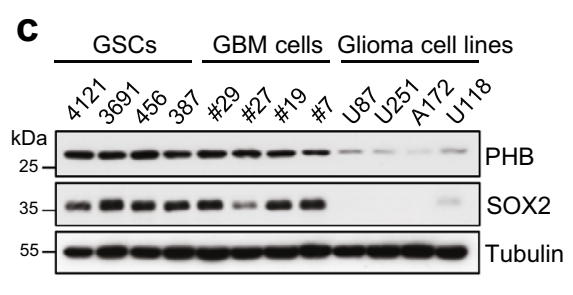

e

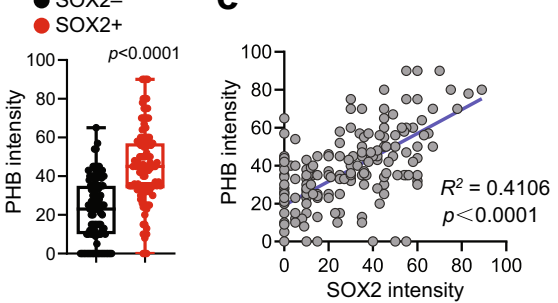

h

GBM\#6
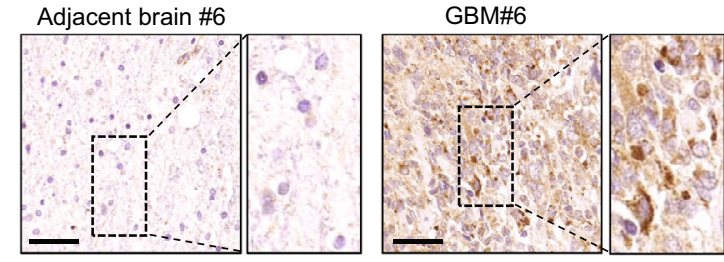

k
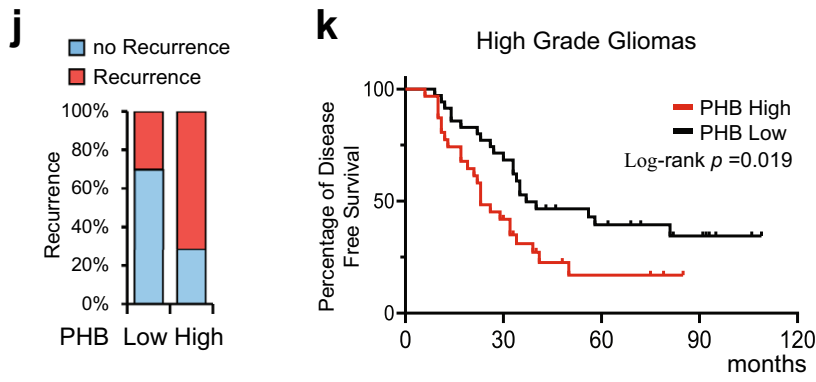

Fig. 1 PHB is highly expressed in GSCs. a-c Immunoblot (IB) showing the expression of indicated proteins in glioma stem-like cells (GSCs) and matched non-stem tumor cells (NSTCs) (a), in GSCs, normal human astrocyte (NHA), and human neural progenitor cells (hNP1, 17231, and 15167) (b), or in GSCs, primary GBM cells and established glioma cell lines (c). d and e Representative immunofluorescent (IF) images of human primary glioblastoma (GBM) specimens stained with anti-PHB (green) and anti-SOX2 (red). Nuclei were counterstained with Hoechst (blue) (d, left). Scale bars, $40 \mu \mathrm{m}$. Quantifications of PHB staining intensity in SOX2+ $(n=110)$ and SOX2 $-(n=100)$ cells (five random microscope fields from three tumors) are shown (d, right). Pearson's correlation coefficient between PHB and SOX2 staining intensity in GBM cells is shown (e). $\mathbf{f}$ mRNA expression of PHB in GSCs ( $n=19)$ relative to bulk tumor cells (BTCs, $n=7$ ) from GEO profile (GSE86237) are shown. $\mathbf{g}$ IB showing the levels of PHB and PHB2 in human primary GBM tissues and adjacent normal brain tissues. $\mathbf{h}$ Immunohistochemical (IHC) staining of PHB in primary GBM and matched adjacent brain tissue. Scale bars, $100 \mu$ m. $\mathbf{i}-\mathbf{k}$ $\mathrm{IHC}$ analysis of PHB in a glioma tissue microarray. Representative images and boxplots of histoscore of PHB in low grade and high grade gliomas are shown (i). Scale bars, $50 \mu \mathrm{m}$. (low grade, $n=94$; high grade, $n=66$ ). The percentages of recurrence of gliomas in tumors with low $(n=79)$ or high $(n=81)$ expression of PHB are shown (j). Kaplan-Meier survival analysis of patients with PHB low $(n=35)$ and PHB high $(n=31)$ expression in high-grade gliomas are shown (k). (Log-rank Mantel-Cox test). See Supplementary Table 1. Boxplots represent the median, 25th, and 75th percentiles. The maximum and minimum are connected to the center box through the vertical lines (whiskers). $\mathbf{d}, \mathbf{f}, \mathbf{i}$ Unpaired two-sided Student's $t$-test (d, $\mathbf{f}$ ), Welch's two-sided $t$-test (i). Source data are provided as Source Data file.

examined the levels of miR-27a in GSCs, matched NSTCs and normal brain cells. In contrast to the high expression of $\mathrm{PHB}$ in GSCs, miR-27a was highly expressed in NSTCs, NHA, and hNP1 (Supplementary Fig. 2b, c). Transduction of GSCs with miR-27a mimics significantly decreased the expression of $P H B$ (Supplementary Fig. 2d, left). However, transduction of the miR-27a inhibitor upregulated the mRNA levels of $P H B$ in GSCs (Supplementary Fig. 2d, right). To further explore whether miR-27a suppresses $P H B$ expression directly through the putative binding site in the $3^{\prime} \mathrm{UTR}$ of $P H B$, we generated luciferase reporter constructs containing the wild-type or mutated miR-27abinding site (Supplementary Fig. 2e). Co-transfection of the wildtype binding site reporter with miR-27a mimic or with miR-27a inhibitor, respectively, decreased or increased luciferase reporter activity (Supplementary Fig. 2f). However, the activity of luciferase reporter with a mutated $P H B$ 3'UTR was not significantly altered by the miR-27a mimic (Supplementary Fig. 2g). Additionally, the repression of miR-27a on $\mathrm{PHB}$ expression was validated at the protein level in GSCs (Supplementary Fig. 2h). These data suggest that miR-27a mediates the high expression of PHB in GSCs.

Taken together, these results demonstrate that PHB is highly expressed in GSCs and associated with the malignant progression of gliomas.

PHB promotes GSC self-renewal and tumor progression. To explore the functional roles of PHB in GSCs, we utilized the CRISPR-Cas9 system to knock out (KO) PHB genes in different 
a

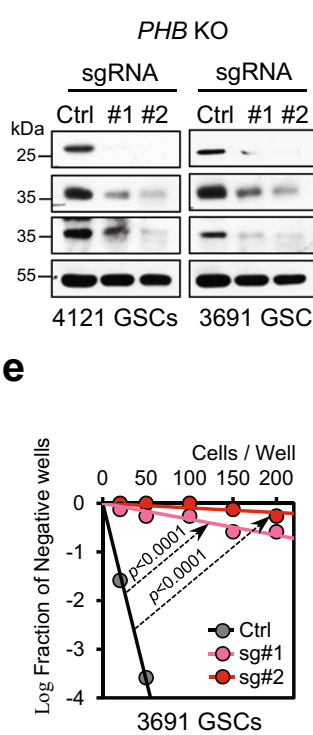

b

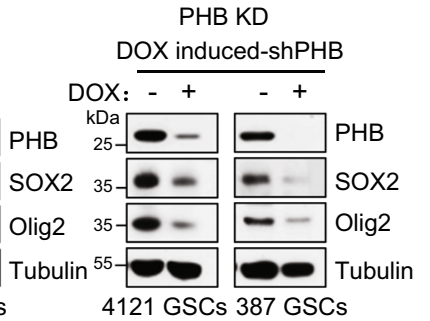

C

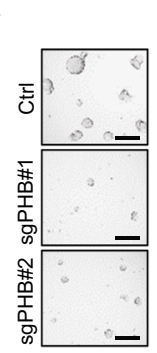

f

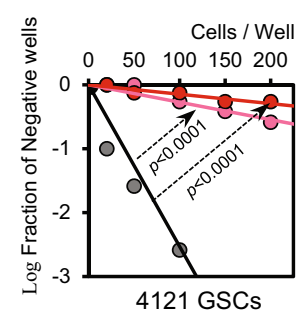

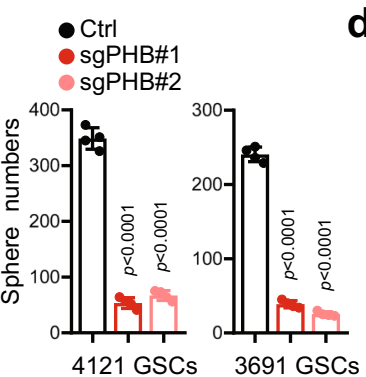

d

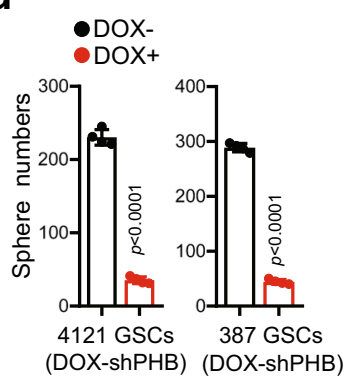

h

4121 xenograft (DOX-shPHB)
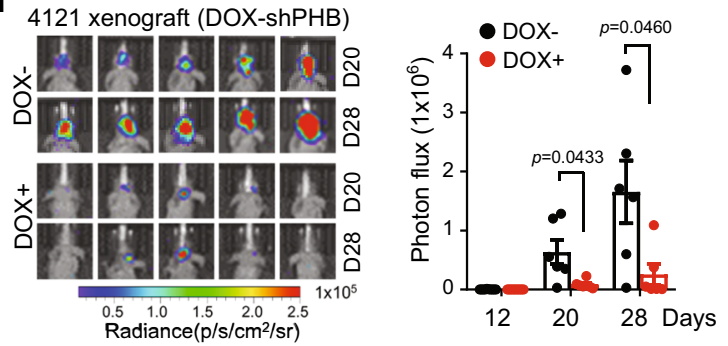

i

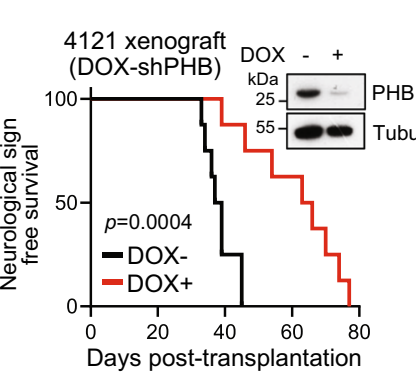

g
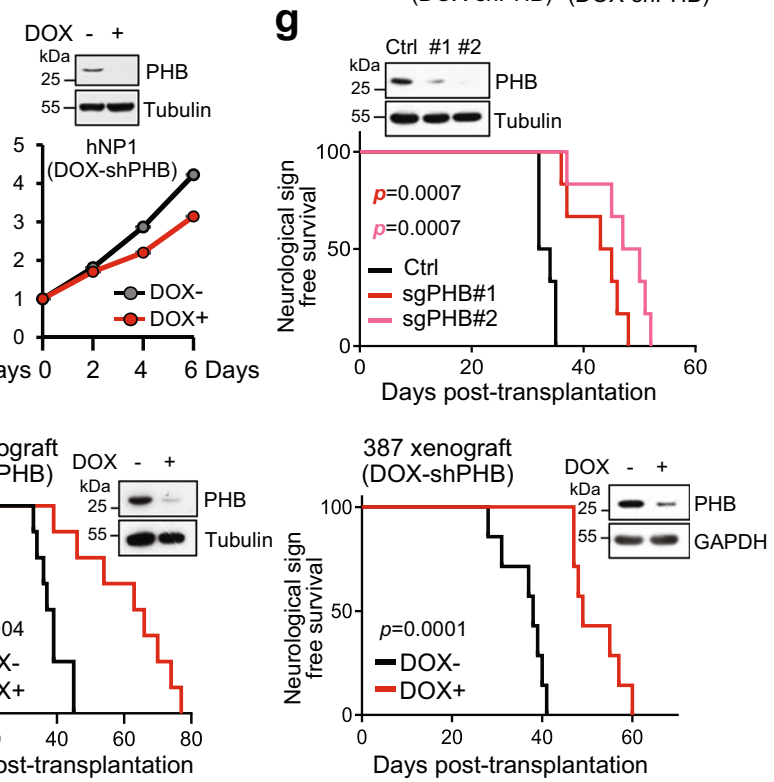

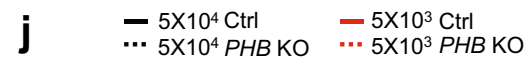

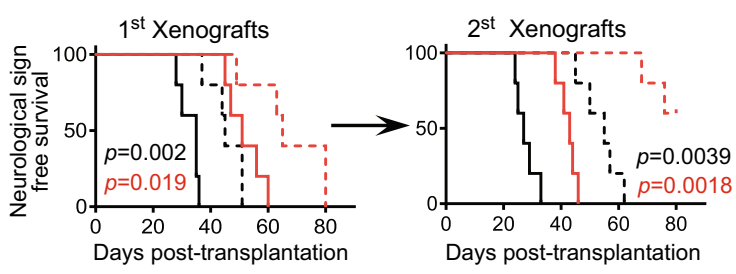

k
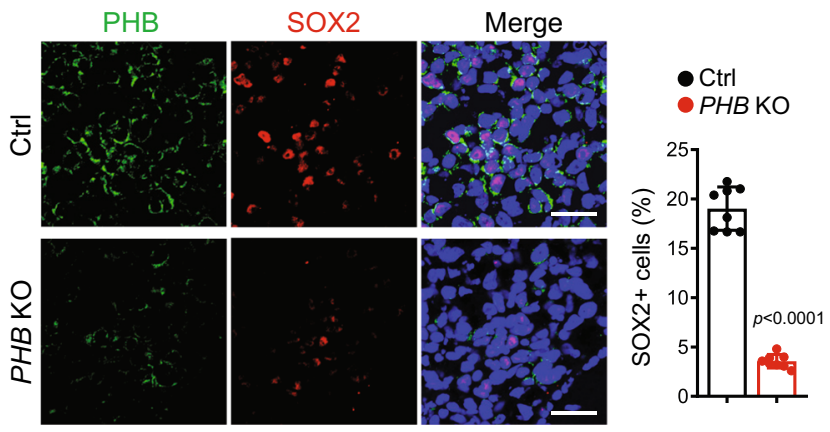

Fig. 2 PHB promotes GSC self-renewal and tumor progression. a PHB knockout GSCs were generated by CRISPR-Cas9 system. IB showing levels of indicated proteins in Ctrl and PHB KO GSCs. b GSCs stably transduced with Tet-on-inducible-shPHB were treated with DOX (100 ng/ml) or vehicle control. Expression of indicated proteins were assessed by IB. c, d Quantifications of tumorsphere numbers (2000 cells/well) formed by Ctrl or PHB KO GSCs (c, right), or control or PHB inducible-KD GSCs (d) (mean \pm SD, $n=4$, biologically independent experiments). Representative images of tumorspheres are shown (c, left). Scale bar, $100 \mu \mathrm{m}$. e In vitro extreme limiting dilution assays (ELDAs) show that PHB KO decreased the frequency of tumorsphere formation in GSCs. $\mathbf{f}$ Inducible-KD of PHB had limit effects on cell growth of NHA (left) and hNP1 (right), as measured by cell viability assay. $\mathbf{g}^{*}$ Ctrl or PHB KO $4121 \mathrm{GSCs}\left(5 \times 10^{4}\right.$ /mouse) were implanted into the brains of nude mice $(\mathrm{nu} / \mathrm{nu}, n=6)$. Kaplan-Meier survival curve of mice is shown (Log-rank Mantel-Cox test). IB showing the efficiency of PHB KO in xenografts (top). $\mathbf{h}$, i GSCs transduced with Tet-on-inducible-shPHB and Luciferase reporter were implanted into the brains of nude mice ( $\mathrm{nu} / \mathrm{nu}$ ). Mice were treated with vehicle control or DOX (2 $\mathrm{mg} / \mathrm{ml}$ in drinking water) to induce expression of shPHB from day 0. GBM xenografts ( $4121 \mathrm{GSCs}$ ) were tracked by bioluminescence (h, left). Bioluminescent quantification of tumor growth is shown (h, right) (mean \pm SEM, $n=6$, biologically independent mice). Kaplan-Meier survival curves of mice are shown (i) (4121 GSCs, $n=8 ; 387$ GSCs, $n=7$; Log-rank Mantel-Cox test). IB showing the efficiency of PHB knockdown in the xenografts (top). $\mathbf{j}$ The in vivo serial transplantation assay shows that PHB KO inhibits GSC self-renewal in vivo. Kaplan-Meier survival curves of mice implanted with indicated GSCs (4121) are shown (top) ( $n=5$; Log-rank Mantel-Cox test). Summary of mice medium survival in the serial transplantation assay is shown (bottom). $\mathbf{k}$ Co-IF staining of PHB (green) and SOX2 (red) in GBM xenografts derived from Ctrl or PHB KO GSCs (4121). Quantifications of SOX2+ cells are shown (right) (mean \pm SD, images $n=8$, from 4 biologically independent samples). Nuclei were counterstained with Hoechst (blue). Scale bars, $40 \mu \mathrm{m}$. Unpaired two-sided Student's $t$-test (c-e, h), Welch's two-sided $t$-test $(\mathbf{k})$. Source data are provided as Source Data file. 
GSC lines. $\mathrm{KO}$ of $\mathrm{PHB}$ using two independent small guide RNAs (sgRNAs) effectively deleted endogenous PHB expression and significantly decreased the levels of SOX2 and Olig2 in GSCs (Fig. 2a and Supplementary Fig. 3a). These results were validated with PHB knockdown (KD) by expressing doxycycline (DOX)inducible small hairpin RNAs (DOX-induced shRNAs) in GSCs (Fig. 2b). The downregulation of SOX2 and Olig2 appeared to occur primarily at the post-transcriptional level, as we did not observe a significant decrease in mRNA expression of SOX2 and Olig2 in PHB-deleted GSCs (Supplementary Fig. 3b). Notably, $\mathrm{KO}$ or inducible-KD of PHB strongly inhibited GSC viability (Supplementary Fig. 3c, d), decreased GSC self-renewal capacity as demonstrated by sphere formation assays (Fig. 2c, d) and in vitro limiting dilution assays (LDA) (Fig. 2e). However, PHB KD did not remarkably affect the growth of NHA, NPCs, or NSTCs (Fig. $2 \mathrm{f}$ and Supplementary Fig. 3e), and this may be due to the limited expression of PHB in these cells. As a control, DOX treatment did not cause any changes in stem cell marker expression or cell growth in control GSCs (Supplementary Fig. 3f).

GSCs possess a strong capacity for self-renewal to seed new tumors $^{9,39}$. To explore the role of PHB in GSC tumor initiation, we established orthotopic GBM xenografts by transplanting control or $P H B$ KO GSCs into brains of immunocompromised mice. When the first mouse developed neurological signs, a subset of mice in each group was sacrificed for histological analysis. Mice in the control group developed large tumors, while smaller tumors were found in mice bearing $P H B \mathrm{KO}$ GSCs (Supplementary Fig. 3g). Consequently, mice implanted with $P H B$ KO GSCs exhibited significantly prolonged survival (Fig. 2g). To modulate PHB expression in vivo, GSCs stably expressing luciferase and DOX-induced PHB shRNAs (Luc/ DOX-shPHB) were intracranially injected into mice, which were then treated with or without DOX from day 0 . Inducible $\mathrm{KD}$ of PHB in vivo markedly inhibited GSC tumorigenesis, as indicated by bioluminescence monitoring of tumor response (Fig. 2h), and prolonged animal survival (Fig. 2i). However, overexpression of PHB did not significantly affect SOX2 expression, tumorsphere formation, or tumorigenesis of NSTCs (Supplementary Fig. 3h, i).

To assess whether the impact of $\mathrm{PHB}$ depletion on tumor growth is due to the inhibition of GSC self-renewal, we performed an in vivo serial transplantation assay, the gold standard for evaluation of CSCs self-renewal ${ }^{6,22,40}$. Briefly, different numbers of GSCs $\left(5 \times 10^{4}\right.$ or $\left.5 \times 10^{3}\right)$ were implanted into the brains of mice. All mice implanted with control GSCs developed tumors in the primary and secondary xenografts. In the $5 \times 10^{4}$ GSCs groups, the difference in median survival between the first transplantation in control and $P H B \mathrm{KO}$ groups was 10 days, which was significantly extended to 28 days upon the 2 nd transplantation (Fig. 2j, black lines). Notably, in the $5 \times 10^{3}$ GSCs groups, $P H B \mathrm{KO}$ prolonged the median survival of mice bearing GSCs to 14 days in the 1 st transplantation. However, only two tumors were observed in mice with the 2nd transplantation of PHB KO GSCs (Fig. 2j, red lines). Meanwhile, we found that both $P H B \mathrm{KO}$ and $\mathrm{PHB}$ inducible-KD resulted in a pronounced reduction of SOX2 + or Olig2 + tumor cells in GBM xenografts (Fig. 2k and Supplementary Fig. 3j, k), suggesting a decrease of GSC pool in PHB-deleted tumors. These results demonstrate that deletion of $\mathrm{PHB}$ compromises GSC self-renewal and tumor progression, suggesting that $\mathrm{PHB}$ could be a specific therapeutic target for GBM treatment.

PHB specifically mediates low levels of mitochondrial ROS in GSCs. To explore the molecular mechanisms by which PHB promotes GSC self-renewal, we first examined the subcellular localization of $\mathrm{PHB}$, as this may be involved in its diverse functions in different tissues $24,25,41$. The ectopically expressed PHBGFP was co-localized with a mitochondrial protein TOM20 in GSCs (Supplementary Fig. 4a). Co-IF staining of PHB and TOM20 or TIM23 (a mitochondrial protein) showed that endogenous $\mathrm{PHB}$ was mainly localized in the mitochondria of GSCs, NSTCs, NPC, and NHA (Supplementary Fig. 4b). Interestingly, we did not observe any change in oxidative phosphorylation (OXPHOS) or ATP generation in mitochondria of PHB-deleted GSCs (Supplementary Fig. 4c, d). However, $P H B$ KO significantly increased peroxide production, as assessed with the redoxsensitive probe $\mathrm{H}_{2}$ DCFDA (Fig. 3a and Supplementary Fig. 4e), and this was further confirmed by PHB inducible-KD in GSCs (Fig. 3b). Redox status plays crucial role in the maintenance and therapeutic resistance of CSCs. Compared to NSTCs, CSCs maintain low levels of ROS and exhibit redox patterns similar to the corresponding normal stem cells ${ }^{15,17,42}$. Consistently, we found that mitochondrial peroxide levels were lower in GSCs than they were in NSTCs (Fig. 3c); however, they were comparable among GSCs, NHA, and NPCs (Fig. 3d). Notably, PHB depletion had no significant effect on the peroxide levels in NHA or NPCs (Fig. 3e). These results reveal that PHB may specifically maintain low levels of mitochondrial ROS in GSCs, suggesting that different mechanisms are involved in ROS regulation between GSCs and NPCs.

To determine whether PHB promotes GSC self-renewal through regulating mitochondrial ROS production, $\mathrm{PHB} \mathrm{KO}$ or PHB inducible-KD GSCs were treated with $\mathrm{N}$-acetyl-L-cysteine (NAC), an ROS-scavenging agent. Treatment with NAC significantly abolished the induction of peroxide by PHB deletion, and this subsequently rescued the reduction of stem cell marker expression and the inhibition of tumorsphere formation due to loss of PHB in GSCs (Fig. 3f and Supplementary Fig. 4f). To further explore the correlation between $\mathrm{PHB}$ expression and cell oxidative stress in vivo, we performed Co-IF staining for PHB and 8-OHdG, a marker of DNA oxidative modification by $\mathrm{ROS}^{43}$, in human GBM specimens. Tumor cells with high PHB expression displayed significantly lower levels of 8-OHdG immunoreactivity, suggesting the suppression of ROS by $\mathrm{PHB}$ (Fig. 3g). Our data also revealed that GSCs contained low levels of ROS in GBM specimens (Supplementary Fig. 4g). The induction of ROS was further confirmed in $\mathrm{PHB} \mathrm{KO}$ and $\mathrm{PHB}$ inducibleKD GBM xenografts (Supplementary Fig. 4h, i).

To understand how mitochondrial ROS induced by PHB loss regulates GSC maintenance, we performed RNA-sequencing analysis in control and $P H B$ KO GSCs. PHB deletion altered (mostly upregulated) the expression of a large number of genes in GSCs (Fig. 3h and Supplementary Table S2). Gene ontology (GO) analysis of the differentially expressed genes revealed that $\mathrm{PHB}$ $\mathrm{KO}$ resulted in a significant upregulation of genes involved in the regulation of cell death and cell differentiation (Fig. $3 \mathrm{i}$ and Supplementary Fig. 4j). Upregulation of genes, such as $A T F 3$, UNC5B, DDIT3, TRIB3, NUPR1 (cell death regulation), INHBE, DDR2 (cell differentiation regulation), ATF3 and ALDH1L2 (responding to oxygen-containing compound stimulus) was confirmed by Q-PCR in $\mathrm{PHB} \mathrm{KO}$ and PHB inducible-KD GSCs (Fig. 3j and Supplementary Fig. 4k). Importantly, the induction of these genes was significantly rescued by NAC treatment in GSCs with $\mathrm{PHB} \mathrm{KO}$ or PHB inducible-KD (Fig. 3k), further supporting that $\mathrm{PHB}$ deletion induces mitochondrial ROS production, that subsequently increases the gene expression signature involved in regulating cell differentiation and cell death. Collectively, our data suggest that the low mitochondrial ROS status in GSCs is specifically mediated by high levels of $\mathrm{PHB}$, which promotes GSC self-renewal. 
a

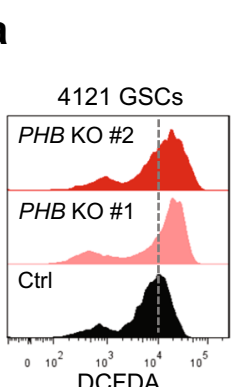

d

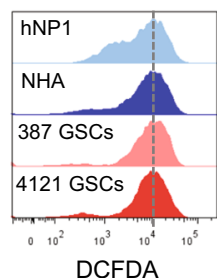

g
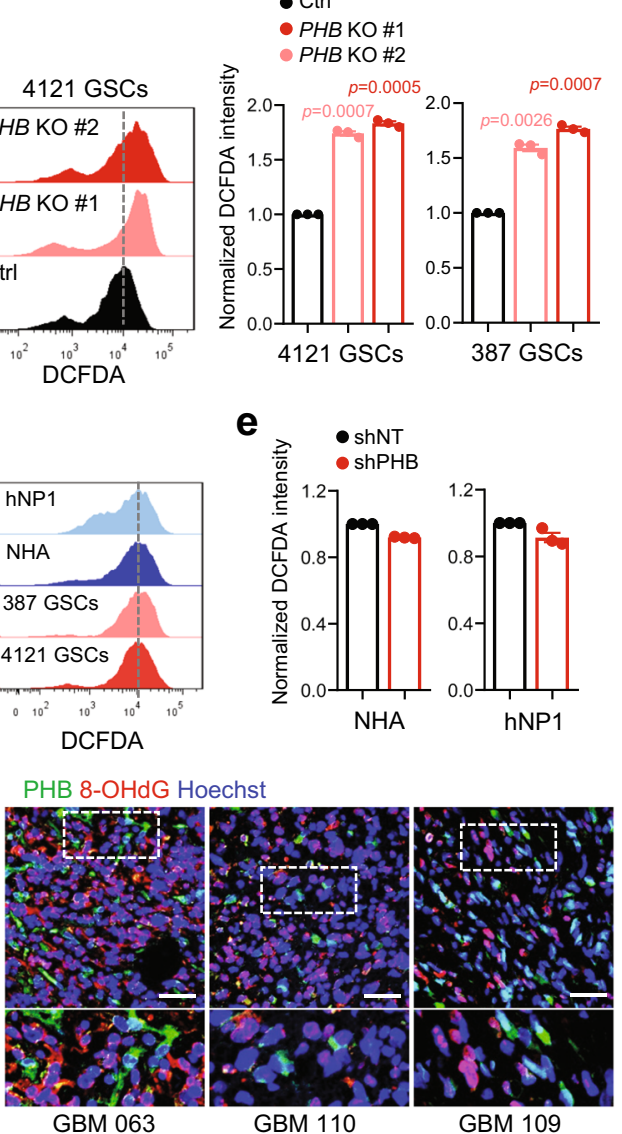

e

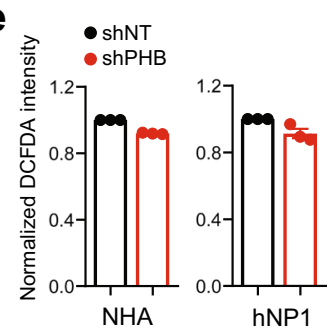

GBM 110 h

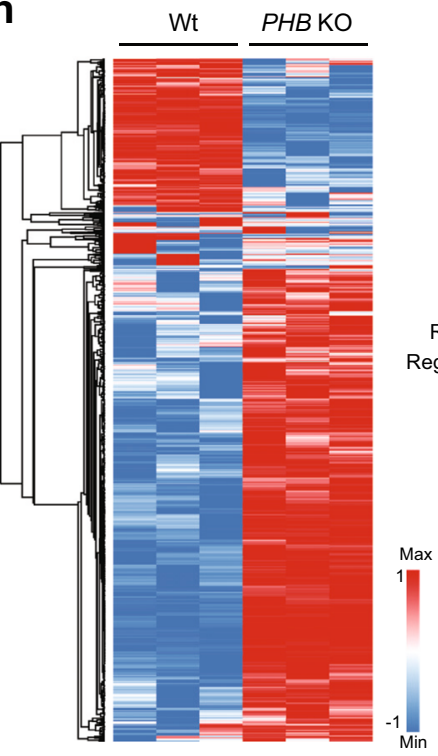

i b

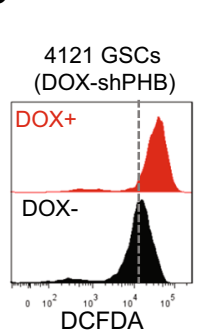

f
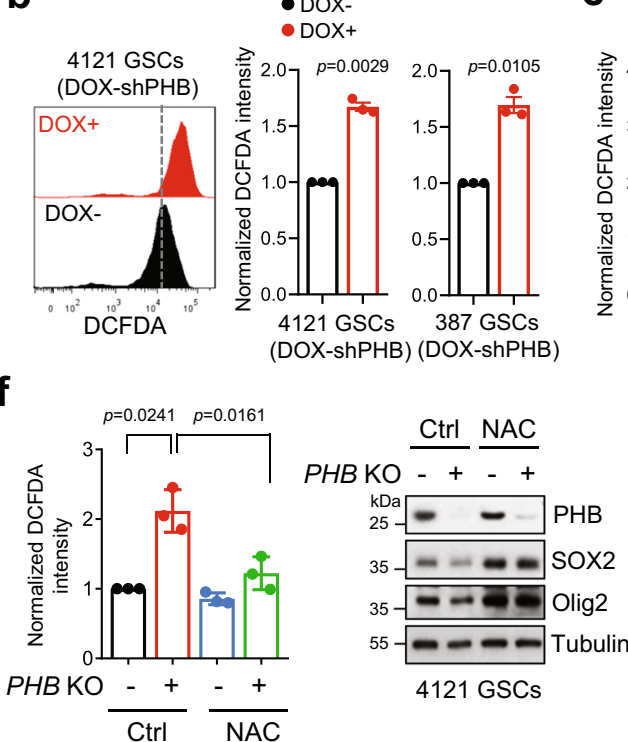

C

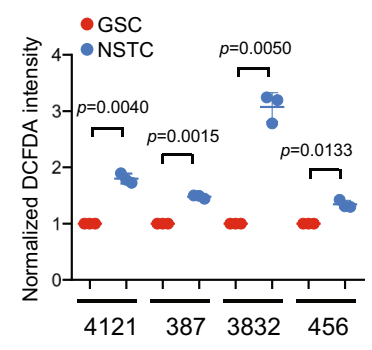

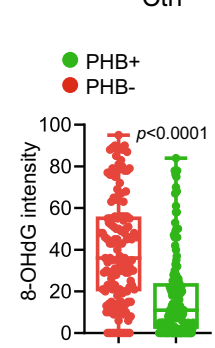

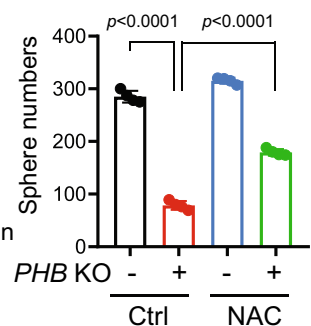

j

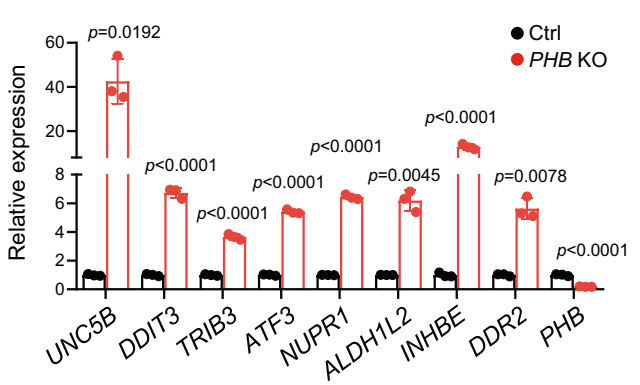

k

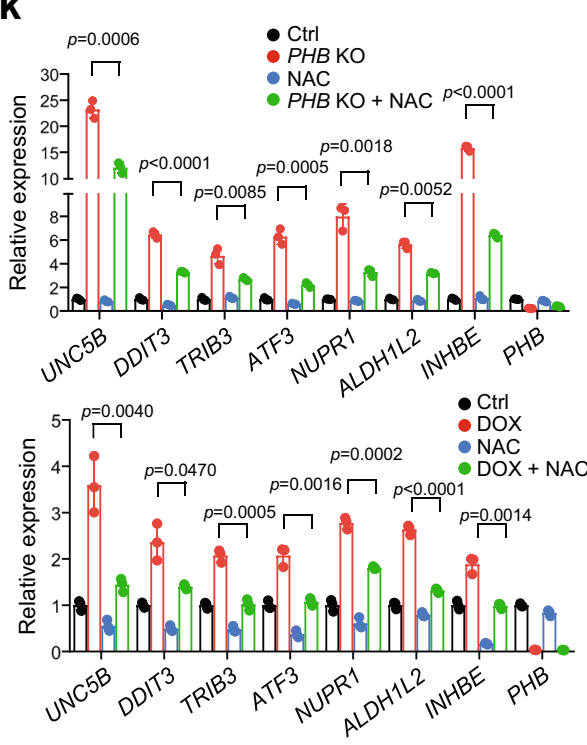

PHB associates with and stabilizes PRDX3 by inhibiting its ubiquitin-proteasome degradation. We next investigated how PHB modulates mitochondrial peroxide production in GSCs. A study suggests that PHB depletion increases ROS levels via the inhibition of mitochondrial complex $\mathrm{I}$ in endothelial cells ${ }^{44}$. However, we did not observe a significant difference in complex I activity between the control and PHB KO GSCs (Supplementary Fig. 5a). Mitochondrial ROS levels are tightly controlled by robust scavenger antioxidant enzymes, such as MnSOD (SOD2), glutathione peroxidase (GPX), and PRDXs ${ }^{13,19,45}$. Thus, we examined whether PHB deletion affects the expression of these scavenger enzymes. $P H B \mathrm{KO}$ remarkably decreased the protein levels of PRDX3, but not those of MnSOD, GPX1, or other PRDX family members such as PRDX1 and PRDX6 in GSCs (Fig. 4a). These results were confirmed by PHB-inducible KD in different GSC lines (Fig. 4b). Moreover, PHB KO exerted no impact on the mRNA expression of PRDX3 (Supplementary Fig. 5b), suggesting that PHB may regulate PRDX3 protein stability. However, PHB depletion slightly decreased PRDX3 levels in NSTC, hNP1, and NHA (Supplementary Fig. 5c). We also assessed other 
Fig. 3 PHB specific mediates low levels of mitochondrial peroxide in GSCs. a-e The peroxide levels, as indicated by DCFDA fluorescence, were measured by flow cytometry in PHB KO GSCs (a), PHB inducible-KD GSCs (b), GSCs and matched NSTCs (c), GSCs, hNP1 and NHA (d), or PHB KD NHA and hNP1 (e) (mean $\pm \mathrm{SD}, n=3$, biologically independent experiments). $\mathbf{f}$ Ctrl or PHB KO $4121 \mathrm{GSC}$ were treated with vehicle control or NAC (5 mM) for $36 \mathrm{~h}$. Flow cytometry analysis of peroxide by DCFDA staining is shown (left). IB of PHB, SOX2, and Olig2 levels are shown (middle). Quantifications of tumorsphere numbers (2000 cells/well) formed by GSCs are shown (right) (mean \pm SD, $n=3$, biologically independent experiments). $\mathbf{g}$ Co-IF staining of PHB (green) and 8-OHdG (red) in primary GBM specimens are shown. Quantifications of 8-OHdG staining intensity in PHB- $(n=139)$ and PHB+ $(n=127)$ cells are shown (right). (Boxplots represent the median, 25th, and 75th percentiles). Nuclei were counterstained with Hoechst (blue). Scale bars, $40 \mu \mathrm{m}$ (up), $20 \mu \mathrm{m}$ (down). $\mathbf{h}$ and $\mathbf{i}$ RNA-seq analysis in control and PHB KO $4121 \mathrm{GSC}$. In $\mathbf{h}$, the heatmap shows relative expression levels of genes downregulated or upregulated in the indicated cells $(p<0.05, F C>2)$. It includes, respectively, 185 and 740 genes downregulated and upregulated in PHB KO compared to control GSCs. Raw data were $\log _{2}$ transformed. A relative color scheme used the minimum and maximum values in each row to convert values to colors. In i, overrepresented Gene Ontology (GO) terms from RNA-seq analysis of upregulated gene sets (top) and downregulated gene sets (bottom) in PHB KO compared to control GSCs. j Quantitative real-time PCR (Q-PCR) analysis of mRNA levels of indicated genes in Ctrl and PHB KO $4121 \mathrm{GSCs}$ (mean \pm SD, $n=3$, biologically independent experiments). $\mathbf{k}$ Q-PCR analysis of mRNA levels of indicated genes in PHB KO (top) and PHB inducible-KD (bottom) 4121 GSCs treated with vehicle control or NAC ( $5 \mathrm{mM})$ (mean $\pm \mathrm{SD}, n=3$, biologically independent experiments). Welch's two-sided $t$-test (a-c, e-g, $\mathbf{j}$, $\mathbf{k}$ ), Unpaired two-sided Student's t-test (f, right). Source data are provided as Source Data file.

antioxidants, including CuZnSOD, GSH, and catalase, and we found that PHB deletion or depletion exerted no significant effect on the levels or activities of these enzymes in GSCs (Supplementary Fig. 5d-f).

PRDX3, a 2-cysteine (Cys) thiol reductase of the PRDX family, is localized in mitochondria. Studies suggest that PRDX3 contributes to nearly $90 \%$ of mitochondrial peroxide elimination ${ }^{19,22}$. We observed that PHB was colocalized with PRDX3 in the mitochondria of GSCs (Supplementary Fig. 5g). To assess whether PHB binds to PRDX3, we performed coimmunoprecipitation (Co-IP) with Flag-PRDX3 3 and found that endogenous PHB interacted with Flag-PRDX3 in GSCs (Fig. 4c). The association between endogenous PRDX3 and PHB was further validated by Co-IP with anti-PHB or anti-PRDX3 antibody (Fig. 4d). PHB was found to interact with its homolog PHB2 (Fig. 4d, left), and this was consistent with a previous report ${ }^{46}$. Interestingly, our results showed that PRDX3 was associated with PHB, but not with PHB2 (Fig. 4c, d), suggesting that different PHB complexes might exist in the mitochondria. We further mapped the interaction regions of these two proteins, and we found that the N-terminal of PRDX3 and the PHB/ Coiled-coil domain of PHB were required for their association (Supplementary Fig. 5h, i).

To determine whether PHB regulates PRDX3 protein stability, we performed cycloheximide (CHX) chase assay to examine the proteolytic turnover of PRDX3 in control and PHB-depleted GSCs. We found that PRDX3 protein remained quite stable, as CHX treatment for $12 \mathrm{~h}$ did not noticeably impact its stability. However, the half-life of PRDX3 protein, but not other PRDX family members such as PRDX1, was significantly decreased in PHB-depleted GSCs (Fig. 4e and Supplementary Fig. 5j). To assess the potential mechanisms associated with PRDX3 degradation, we treated GSCs with MG132, an inhibitor of the proteasome, or chloroquine, an inhibitor of lysosomal acidification. The results showed that treatment with MG132, but not chloroquine, increased the protein levels of PRDX3 and PHB in a time-dependent manner (Supplementary Fig. 5k). Notably, MG132 treatment largely rescued the decrease in PRDX3 protein in $\mathrm{PHB} \mathrm{KO}$ or PHB inducible-KD GSCs (Fig. $4 \mathrm{f}$ ), suggesting that the ubiquitin-proteasome pathway may be required for PRDX3 reduction induced by PHB deletion. Indeed, the Lys 48-linked poly-ubiquitination and total poly-ubiquitination of PRDX3 were dramatically increased in GSCs with PHB inducible-KD (Fig. 4g). Overexpressing PHB resulted in a decrease in the Lys 48-linked poly-ubiquitination and total poly-ubiquitination of PRDX3 (Fig. 4h). These data suggest that PHB promotes the stability of PRDX3 protein through the ubiquitin-proteasome pathway.
We next tested whether the functions of PHB are mediated by PRDX3 in GSCs. KD of PRDX3 decreased SOX2 and Olig2 expression, strongly induced peroxide levels, and inhibited cell growth in GSCs (Supplementary Fig. 5l-n). Importantly, overexpression of PRDX3 largely rescued the induction of peroxide and the inhibition of cell growth by PHB depletion in GSCs (Fig. 4i and Supplementary Fig. 5o). Additionally, the induction of indicated gene expression caused by PHB depletion was also significantly rescued by PRDX3 overexpression (Fig. 4j), supporting the crucial role of PRDX3 in PHB signaling. We also confirmed that PHB deletion decreased PRDX3 levels in GSCderived xenografts (Supplementary Fig. 5p). Collectively, these results suggest that PHB maintains low levels of mitochondrial ROS through binding to PRDX3 and suppressing its ubiquitin-proteasome degradation in GSCs (Fig. 4k).

PHB promotes GSC radio-resistance. It is well recognized that ROS is a critical mediator of IR-induced cell killing. IR directly causes DNA double-strand breaks or indirectly induces DNA damage by increasing ROS production ${ }^{16,47}$. To investigate whether the ROS induced by PHB loss sensitizes GBM to radiotherapy, we established the orthotopic GBM xenografts using GSCs expressing Luc/DOX-shPHB. When tumors reached a similar size (day 19 after 4121 GSCs transplantation, or day 14 after 387 GSCs transplantation) (Fig. 5a, c, up), mice were grouped randomly and treated with control, IR (3 Gy, once every week, four times in total), DOX (daily in water) or IR plus DOX (Fig. 5a, c, up). The results showed that abrogating PHB expression potently inhibited tumor growth in the established GBM models (Fig. 5a, c) and extended survival (Fig. 5b, d). IR monotherapy showed limited (Fig. 5a, b) or effective (Fig. 5c, d) suppressive effects on the growth of the established tumors and slightly extended survival. Importantly, losing PHB expression significantly sensitized tumors to IR treatment (Fig. 5a, c) and conferred the longest survival extension among all experimental groups (Fig. 5b, d), highlighting targeting PHB as a therapeutic index for GBM combination therapy. In contrast, we did not observe a significant impact of DOX treatment on the sensitivity of GSCs to IR in vitro or in vivo (Supplementary Fig. 6a, b).

TUNEL or cleaved-caspase3 staining showed that, whereas suppression of PHB expression or IR treatment alone increased tumor cell apoptosis in vivo, the combination treatment resulted in much more cell death in tumors (Fig. 5e), confirming that PHB depletion sensitizes GBM to IR treatment. In addition, IR treatment-induced peroxide production in GSCs in vitro, which was significantly increased by combination with PHB deletion (Fig. $5 \mathrm{f}$ and Supplementary Fig. 6c). However, overexpression of Flag-PHB decreased the accumulation of peroxide-induced by IR 


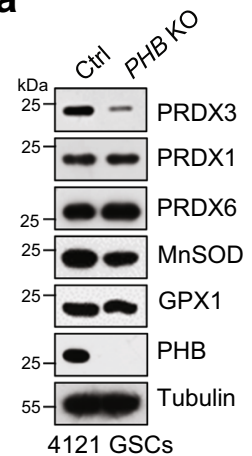

b

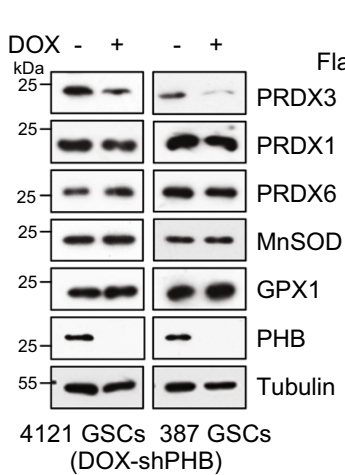

e (DOX-shPHB)
C

Flag-PRDX3 $\frac{\text { Input }}{-+} \frac{\text { IP: Flag }}{-+}$
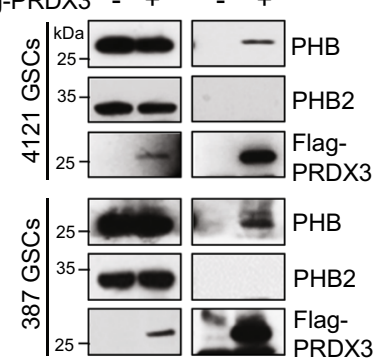

d

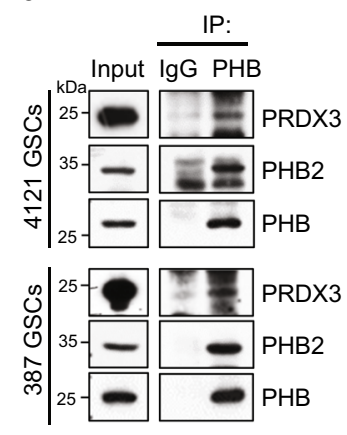

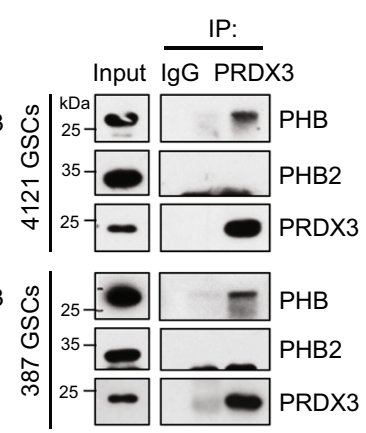

i
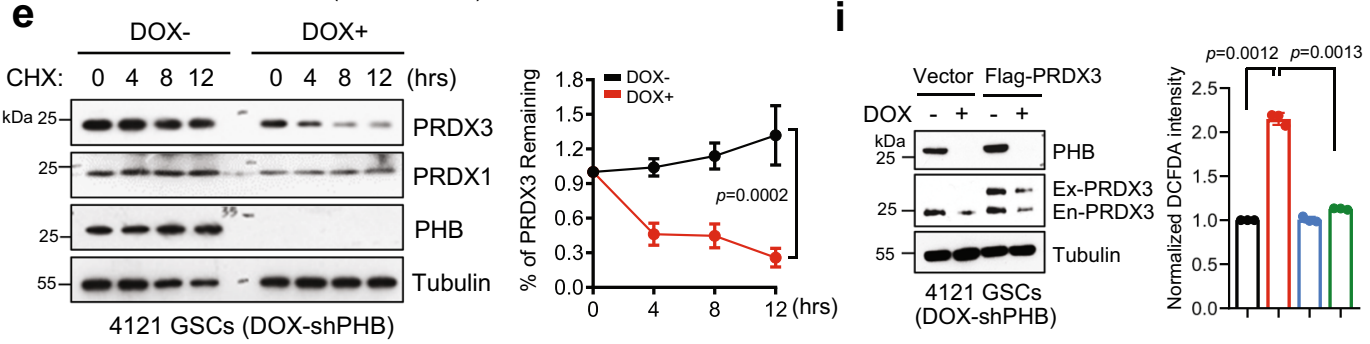

- Ctrl

DOX

- Flag-PRDX3

- DOX + Flag-PRDX3

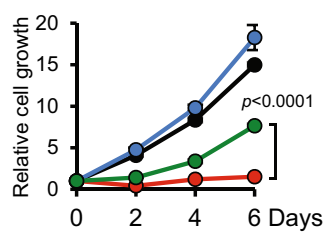

f

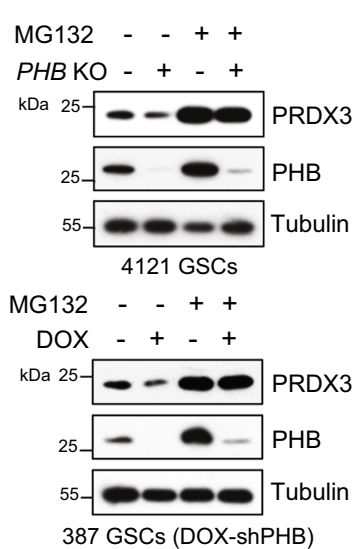

387 GSCs (DOX-shPHB) g
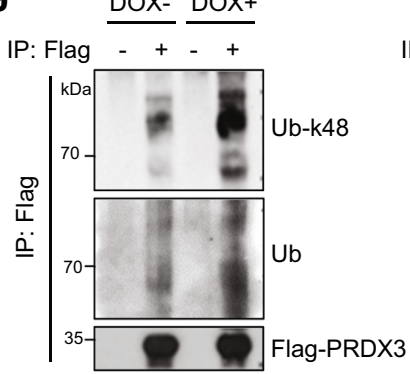

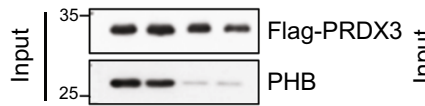
4121 GSCs (DOX-shPHB)

IP: Flag $\frac{\text { DOX- }}{-++} \frac{\mathrm{DOX}+}{-+}$
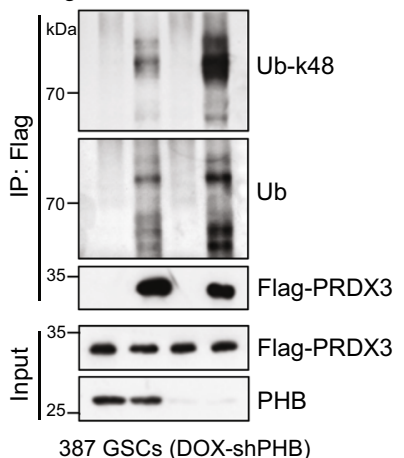

PHB Wt

k

k

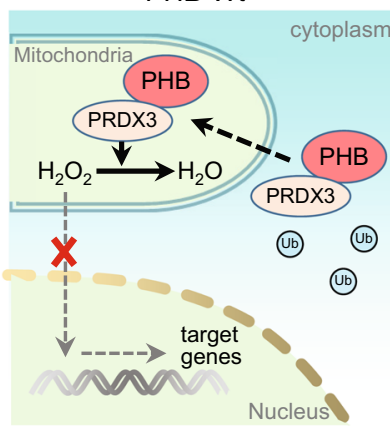

GSC Self-renewal \& Radioresistance h IP: Flag $\frac{\text { Vector }}{-+++}$

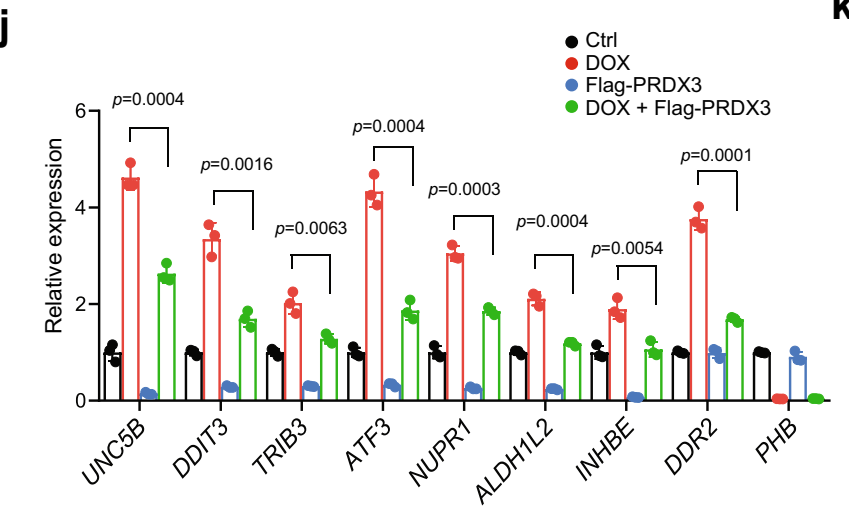

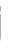

in GSCs (Fig. 5g and Supplementary Fig. 6d). Furthermore, abrogation of PHB remarkably increased GSC apoptosis induced by IR in vitro, as evaluated by flow cytometry analyses of Annexin $\mathrm{V}$ and propidium iodide (PI) (Fig. 5h and Supplementary Fig. 6e, left), or by IB analysis with cleaved-caspase 3 and cleaved-PARP (Fig. 5i and Supplementary Fig. 6f, left). Overexpression of FlagPHB strongly decreased GSC apoptosis induced by IR (Fig. 5h, i, and Supplementary Fig. 6e, f, right). Notably, the synergistic effect of $P H B \mathrm{KO}$ and IR on peroxide induction and cell killing was partially rescued by NAC treatment in vitro (Supplementary Fig. 6g, h).

We observed that IR treatment resulted in an increase of DNA damage in GSCs, as assessed by phospho-histone 2A.X ( $\gamma \mathrm{H} 2 \mathrm{AX})$, a marker of DNA double-strand breaks (Supplementary Fig. 6i, left). $P H B \mathrm{KO}$ elevated the $\gamma \mathrm{H} 2 \mathrm{AX}$ levels even in the absence of IR treatment, suggesting the induction of DNA damage by $\mathrm{PHB}$ 
Fig. 4 PHB associates with and stabilizes PRDX3 by inhibiting its ubiquitin-proteasome degradation. $\mathbf{a}$, $\mathbf{b}$ IB showing levels of indicated proteins in GSCS with PHB KO (a) or PHB inducible-KD (DOX, $100 \mathrm{ng} / \mathrm{ml}$ ) (b). c Co-immunoprecipitation (Co-IP) with anti-Flag M2 beads in Flag-vector and Flag-PRDX3 expressing GSCs and IB for PHB, PHB2, and PRDX3 are shown. d Co-IP with anti-PHB (left) or anti-PRDX3 (right) antibody in GSCs and IB for PHB, PHB2, and PRDX3 are shown. Immunoglobulin G ( $\mathrm{gG}$ ) was used as a control antibody for IPs. e IB showing the CHX ( $50 \mu \mathrm{g} / \mathrm{ml}) \mathrm{chase}$ analysis of PRDX3 protein degradation at indicated time points in GSCs with or without PHB inducible-KD (DOX, $100 \mathrm{ng} / \mathrm{ml}$ ). Quantifications of relative protein levels of PRDX3 are shown (right) (mean $\pm S E M, n=5(0,4,8 \mathrm{~h})$ or $n=3(12 \mathrm{~h})$, biologically independent experiments, Two-way ANOVA). $\mathbf{f} I \mathrm{~B}$ showing the levels of PRDX3 and PHB in PHB KO (top) or PHB inducible-KD GSCs (bottom) treated with vehicle control or MG132 (10 $\mu \mathrm{M})$ for $12 \mathrm{~h}$. $\mathbf{g}$ Flag-PRDX3 expressing GSCs with or without PHB inducible-KD (DOX, $100 \mathrm{ng} / \mathrm{ml})$ were treated with MG132 $(10 \mu \mathrm{M})$ for $12 \mathrm{~h}$. Cell lysates were immunoprecipitated with anti-Flag antibody and IB with anti-ubiquitin-Lys48 (Ub-k48) or anti-ubiquitin (Ub) antibody. $\mathbf{h}$ IB showing that overexpression of PHB inhibits ubiquitination of PRDX3 in GSCs (4121). i Ectopic expression of Flag-PRDX3 rescued the induction of peroxide levels and the inhibition of cell growth by PHB depletion in GSCs. IB showing the levels of PRDX3 and PHB in 4121 GSCs (left). The peroxide levels, as indicated by DCFDA fluorescence, were measured by flow cytometry (middle, mean $\pm \mathrm{SD}, n=3$, biologically independent experiments, Welch's two-sided $t$-test). Cell growth of GSCs was assessed by cell viability assay (right, $n=3$, biologically independent experiments, two-way ANOVA). $\mathbf{j}$ Q-PCR analysis of mRNA levels of indicated genes in Ctrl or PHB inducible-KD GSCs (4121) with Flag-vector or Flag-PRDX3 overexpression (mean $\pm S D, n=3$, biologically independent experiments, unpaired two-sided Student's $t$-test). k Proposed model for PHB-PRDX3-mediated regulation of mitochondrial ROS homeostasis. In GSCs, PHB is highly expressed, associated with and stabilizes PRDX3 to maintain mitochondrial ROS homeostasis. Loss of PHB increases PRDX3 ubiquitin-proteasome degradation, elevates ROS levels, and subsequently triggers gene expression to induce GSC differentiation and death. Source data are provided as Source Data file.

deletion. Importantly, PHB deletion strongly increased, and FlagPHB overexpression decreased the DNA damage induced by IR (Supplementary Fig. 6i). Moreover, depletion of PHB compromised the repair of DNA damage induced by IR in GSCs (Supplementary Fig. 6j), and this may contribute to the sensitization of GSCs to radiotherapy. In addition to radiotherapy, chemotherapy using the DNA alkylating agent temozolomide (TMZ) is another standard treatment for GBM 4 . Our data showed that PHB depletion also increased the sensitivity of GSCs to TMZ treatment; however, the synergistic effect was weaker than that of the combined treatment of PHB depletion and IR (Supplementary Fig. 6k). Taken together, these data suggest that high levels of PHB protect GSCs from IR-induced cell death through reducing ROS accumulation and that abrogating $\mathrm{PHB}$ synergizes with radiation to improve GBM treatment.

Pharmacological targeting of PHB inhibits GSC self-renewal and sensitizes GBM to radiotherapy. Previous studies have shown that rocaglamide A ( $\operatorname{Roc} A)$, a compound isolated from the genus Aglaia, targets PHB directly by blocking the interaction between $\mathrm{PHB}$ and its binding proteins ${ }^{48,49}$. Consistent with this, we found that RocA bound to endogenous PHB in GSCs (Fig. 6a) and efficiently blocked the association between PHB and PRDX3 in GSCs (Fig. 6b and Supplementary Fig. 7a). Consequently, the expression of PRDX3 and stem cell markers was decreased upon RocA treatment in a time-dependent and dose-dependent manner (Fig. 6c and Supplementary Fig. 7b). We then tested the effect of RocA on GSC maintenance, and we found that RocA treatment caused a dose-dependent inhibition of cell growth in multiple GSC lines (Supplementary Fig. 7c). The half-maximal inhibitory concentration $\left(\mathrm{IC}_{50}\right)$ analysis showed that a very low concentration of RocA $(<5 \mathrm{nM})$ achieved significant suppression of GSC growth (Fig. 6d). However, RocA treatment showed relatively low toxic effects on NHA, NPCs, NSTCs, and the established glioma cell lines at the effective dose for GSCs (Supplementary Fig. 7d-f).

To determine whether the impact of RocA on GSCs is mediated through inhibition of PHB-PRDX3 signaling, we treated control and PHB-inducible KD GSCs with RocA. RocA treatment strongly induced peroxide production in a dose-dependent manner in GSCs (Fig. 6e, g, left). However, RocA did not increase peroxide levels in PHB-depleted GSCs as much as it did in control GSCs (Fig. 6e), suggesting an important role of PHB in ROS induction by RocA. Notably, scavenging peroxide by NAC significantly rescued the inhibition of GSC growth by RocA (Fig. 6f). As RocA treatment resulted in the dissociation of PRDX3 from PHB and a subsequent decrease in PRDX3 protein levels (Fig. 6b, c and Supplementary Fig. 7a, b), we ectopically expressed Flag-PRDX3 in GSCs, and we found that overexpression of PRDX3 potently compromised the induction of peroxide by RocA (Fig. 6g), and largely rescued the inhibitory effect of RocA on GSC growth (Fig. 6h).

Studies suggest that some potential targets, such as NF- $\kappa \mathrm{B}, \mathrm{c}$ Raf, and eIF4A may be involved in the anti-cancer activity of RocA $^{48,50,51}$. We further assessed the effects of RocA on these signaling pathways in GSCs. Our results showed that RocA treatment at the same concentration as that for growth inhibition in GSCs caused no obvious impact on the activation of NF- $\kappa$ B and c-Raf-ERK signaling pathways (Supplementary Fig. 7g, h). Many transcriptional factors and oncogenes, including $\mathrm{c}-\mathrm{Myc}$, $\mathrm{EZH} 2$, Notch1, and $\mathrm{Bcl} 2$, require eIF4A for translation ${ }^{52}$ (Supplementary Fig. 7i, left). However, RocA treatment did not decrease these proteins in GSCs (Supplementary Fig. 7i, right). Together, our results suggest that RocA induces mitochondrial ROS levels and suppresses GSC growth mainly through inhibiting the PHB-PRDX3 pathway. We further investigated the effect of fluorizoline, a synthetic molecule that binds to and targets $\mathrm{PHB}^{53}$, on GSCs. Consistent with the effect of RocA on GSCs, fluorizoline treatment decreased the expression of PRDX3 and stem cell markers in GSCs (Supplementary Fig. 7j), and inhibited GSC growth in a dose-dependent manner (Supplementary Fig. 7k, left). However, fluorizoline also showed a remarkable suppression on hNP1 and NHA (Supplementary Fig. 7k, right), indicating that the undefined targets of fluorizoline may exist. Moreover, treatment with fluorizoline significantly inhibited the established GBM tumor growth and extended survival (Supplementary Fig. 7l).

To justify the RocA use in GBM treatment in vivo, we intraperitoneally injected RocA into normal mice or mice bearing orthotopic GBM xenografts and assessed its bioavailability in mouse brains. The mass spectrometry analysis showed that the concentration of RocA was significantly increased in mouse brains and was higher in brain tumor tissues than that was in normal brains (Supplementary Fig. 8a, b), suggesting that RocA could penetrate mouse brain tumors to exert tumoricidal effects. Importantly, RocA administration remarkably suppressed the growth of the established tumors (Fig. 6i) and conferred a significant survival benefit (Fig. 6j). Moreover, the inhibitory effects of RocA on GSC-derived xenografts were rescued by overexpressing PRDX3 in GSCs (Supplementary Fig. 8c). Consistent with the results from $\mathrm{PHB}$ deletion in GSC-derived xenografts, RocA treatment resulted in a dramatic increase in ROS levels, a reduction in SOX2+ tumor cells, and an increase in 
a

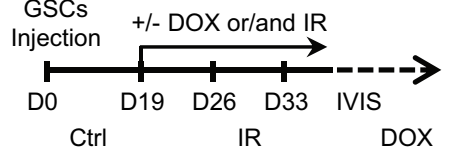

b
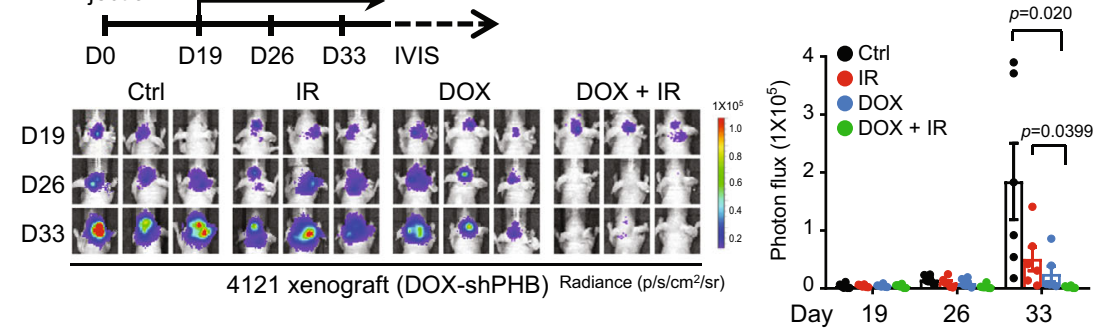

C
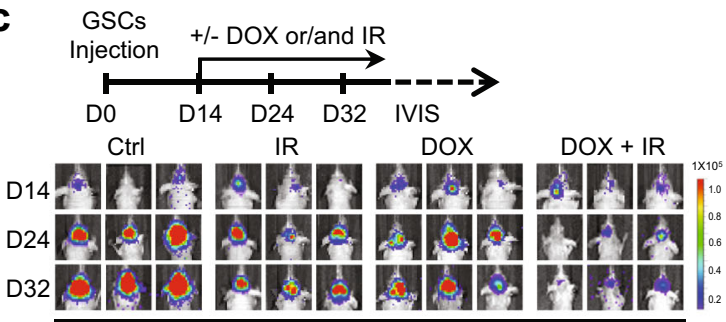

387 xenograft (DOX-shPHB) Radiance $\left(\mathrm{p} / \mathrm{s} / \mathrm{cm}^{2} / \mathrm{sr}\right)$

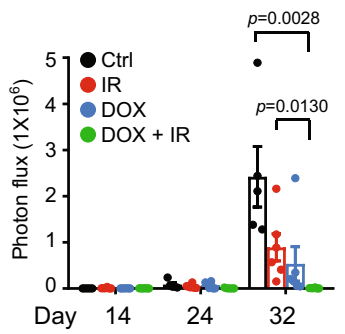

d
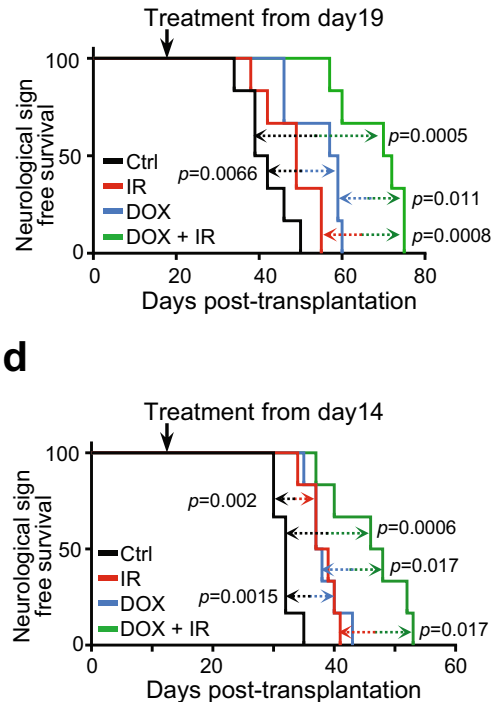

f

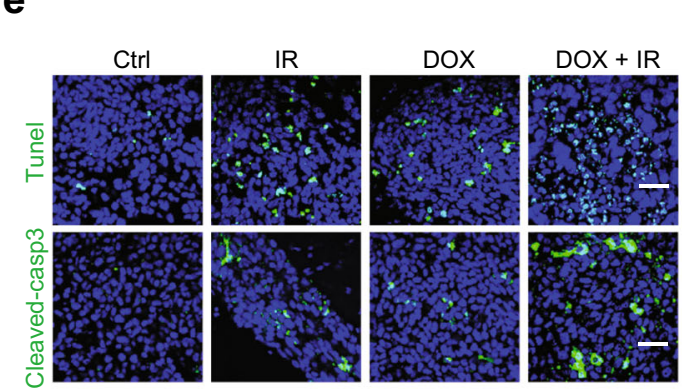

387 GSCs (DOX-shPHB) / Xenograft

h

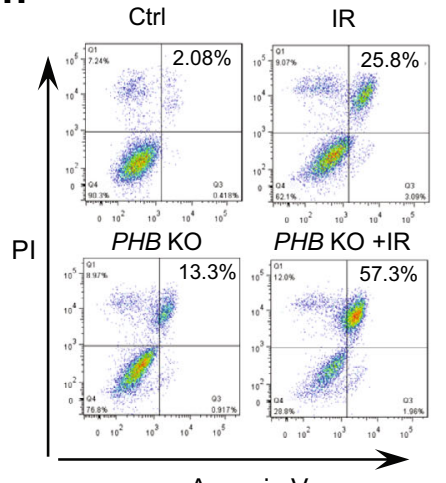

Annexin V

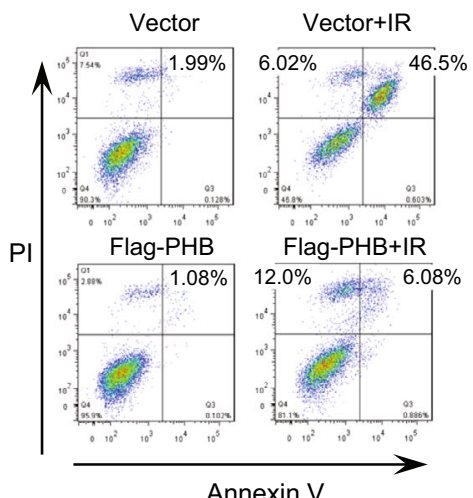

Annexin V
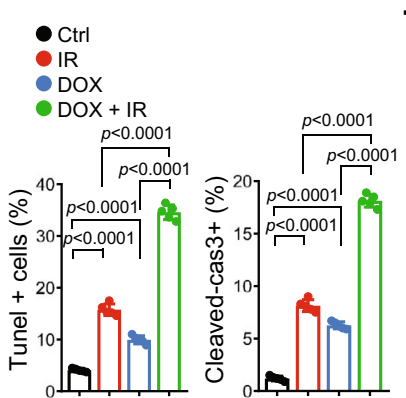

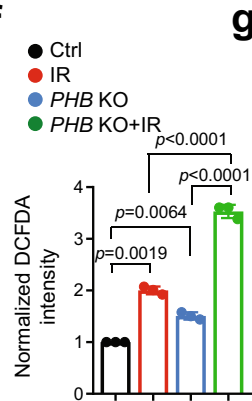

4121 GSCs g

$$
\text { - vector }
$$

IR

Flag-PHB

- Flag-PHB + IR

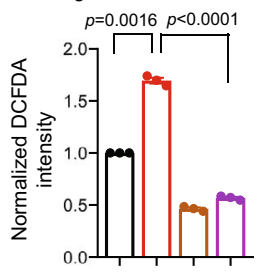

4121 GSCs

i

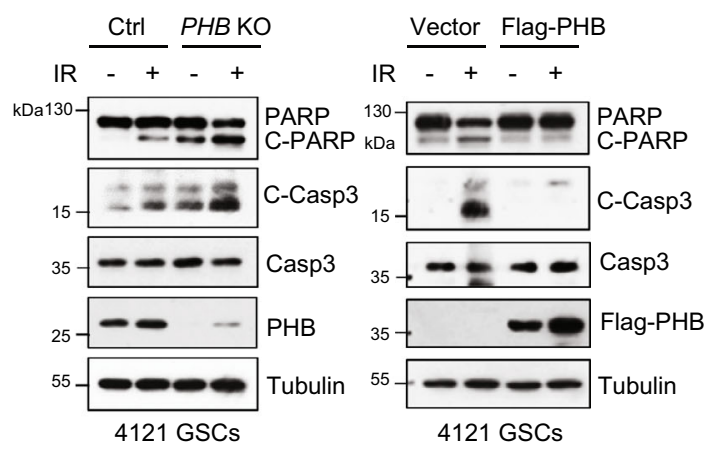

Fig. 5 PHB promotes GSC radio-resistance. a-d Nude mice (nu/nu) were intracranially implanted with GSCs (a, b, 4121 GSCs; c, d 387 GSCs) transduced with Luciferase/Tet-on-inducible-shPHB. Mice were randomly grouped ( $n=6$ for each group) and treated with control, IR ( $3 \mathrm{~Gy}$, once a week, 4 times), DOX $(2 \mathrm{mg} / \mathrm{ml}$ in drinking water), or the combined treatment from day $19(\mathbf{a}, \mathbf{b})$ or day 14 (c, d) after implantation, as shown by schematic representation (a, c top). GBM xenografts were tracked by bioluminescence and the representative images are shown (a, $\mathbf{c}$ bottom). Bioluminescent quantifications of tumor growth are shown (a, c right, mean \pm SEM, unpaired two-sided Student's t-test). Kaplan-Meier survival plots of mice are shown (b, d; Log-rank Mantel-Cox test). e IF staining of Tunel (top) or cleaved-caspase3 (bottom) in GBM xenografts from (c) are shown (left). Quantifications of Tunel+ or cleaved-caspase3+ cells are shown (right) (mean $\pm S D, n=5$, biologically independent samples, Unpaired two-sided Student's $t$-test). Scale bars, $40 \mu m$. $\mathbf{f}, \mathbf{g}$ The peroxide levels, as indicated by DCFDA fluorescence, were measured by flow cytometry in $4121 \mathrm{GSCs}$ with indicated treatments. IR, $3 \mathrm{~Gy}$ for $48 \mathrm{~h}$ (f) or $72 \mathrm{~h}(\mathbf{g})$ (mean $\pm \mathrm{SD}, n=3$, biologically independent experiments, Welch's two-sided $t$-test). $\mathbf{h}$ Cell apoptosis was measured by flow cytometry in $4121 \mathrm{GSCs}$ with indicated treatments. IR, $3 \mathrm{~Gy}$ for $48 \mathrm{~h}$ (left) or $72 \mathrm{~h}$ (right). i IB showing levels of cleaved-PARP, cleaved-caspaspe3, caspase3, and PHB in GSCs with indicated treatments. IR, 3 Gy for $48 \mathrm{~h}$ (left) or $72 \mathrm{~h}$ (right). Source data are provided as Source Data file. 
a

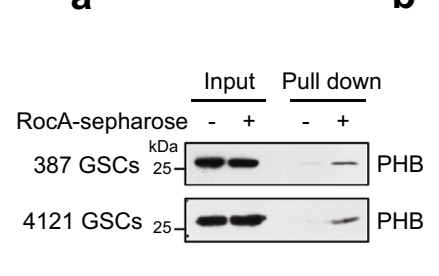

b

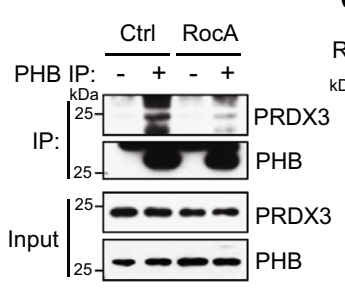

C

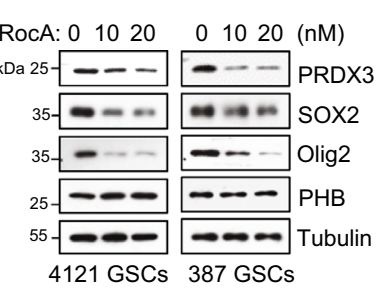

d

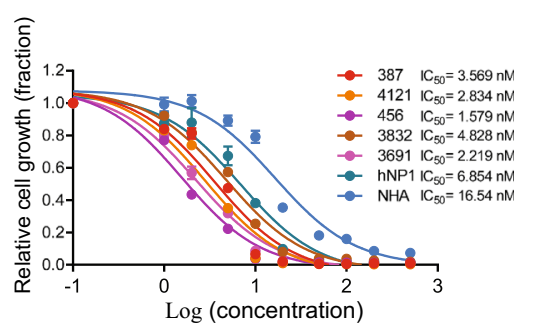

e
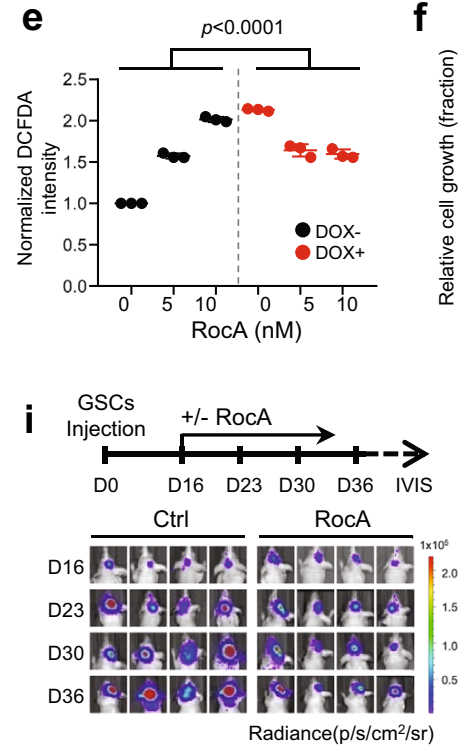

f

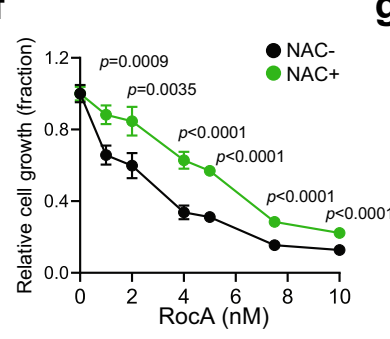

g

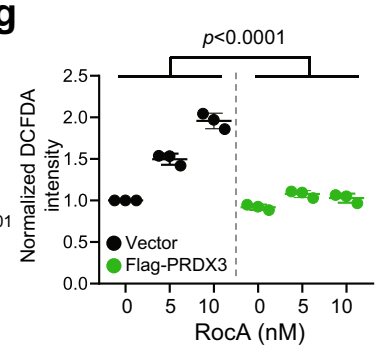

h

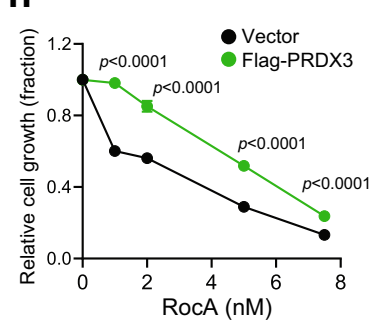

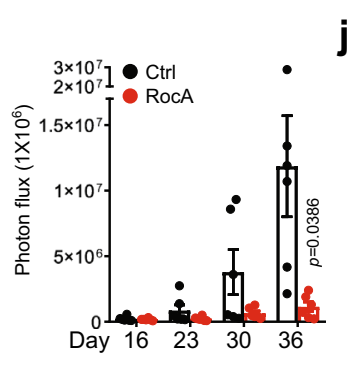
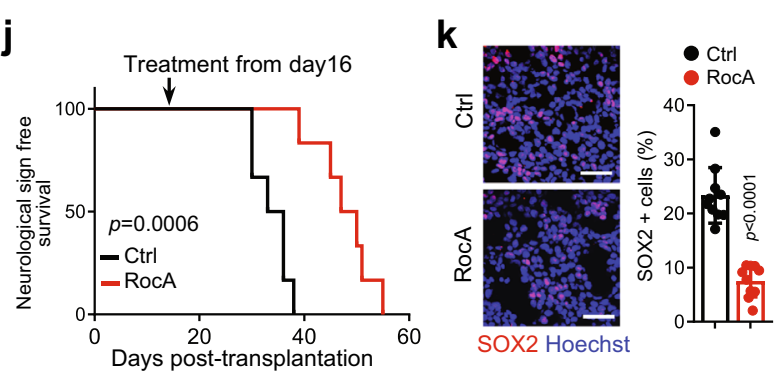

Fig. 6 Pharmacological targeting PHB inhibits GSC growth and tumorigenesis. a The association of RocA and PHB was analyzed by RocA-conjugated sepharose pull-down in GSCs. b Co-IP of PHB in 4121 GSCs treated with RocA (10 nM) and MG132 (10 $\mu$ M) for $12 \mathrm{~h}$ and IB for PHB and PRDX3 are shown. c IB showing the levels of indicated proteins in GSCs treated with increasing dose of RocA for $12 \mathrm{~h}$. $\mathbf{d}$ Dose-response curves of RocA treatment in multiple GSC lines, hNP1 and NHA. Cells were treated with increasing dose of RocA for $48 \mathrm{~h}$. IC $\mathrm{C}_{50}$ values of RocA were measured using nonlinear regression analysis of dose-response curves (mean $\pm \mathrm{SD}, n=3$, biologically independent experiments). $\mathbf{e}, \mathbf{g}$ The peroxide levels, as indicated by DCFDA fluorescence, were measured by flow cytometry in Ctrl or PHB inducible-KD $4121 \mathrm{GSCs}$ (DOX, $100 \mathrm{ng} / \mathrm{ml}$ ) (e), or in Flag-vector or Flag-PRDX3 expressed 4121 GSCs (g) treated with increasing dose of RocA for $18 \mathrm{~h}$ (mean $\pm \mathrm{SD}, n=3$, biologically independent experiments, two-way ANOVA). f, h $4121 \mathrm{GSCs}$ cultured with or without $5 \mathrm{mM} \mathrm{NAC}(\mathbf{f})$, or expressed with Flag-vector or Flag-PRDX3 (h), were treated with indicated doses of RocA for 3 days. Cell growth was assessed by cell viability assay. Data were normalized to the untreated cells of each group (mean \pm SD, $n=4$ (f) or $n=3$ (h), biologically independent experiments, unpaired two-sided Student's $t$-test, no adjustment). i-k Nude mice (nu/nu) intracranially implanted with 4121 GSCs (Luciferase) were randomly grouped $(n=6)$ at day 16 and treated with or without RocA ( $2.5 \mathrm{mg} / \mathrm{kg}$, every 3 days, 8 times in total), as shown by schematic representation (i, top). GBM xenografts were tracked by bioluminescence and the representative images are shown (i, left). Bioluminescent quantification of tumor growth is shown (i, right; mean \pm SEM, Welch's two-sided $t$-test). Kaplan-Meier survival plot of mice is shown (j, Log-rank Mantel-Cox test). IF staining of SOX2 in GBM xenografts with indicated treatments and the representative images are shown (k, left). Quantifications of SOX $2+$ cells are shown (k, right) (mean \pm $S D$, images $n=10$, from five biologically independent samples, Unpaired two-sided Student's t-test, no adjustment). Nuclei were counterstained with Hoechst (blue). Scale bars, $40 \mu \mathrm{m}$. Source data are provided as Source Data file.

cell death in tumors (Fig. 6k and Supplementary Fig. 8d). Notably, RocA treatment did not show detectable toxic effects in vivo, as it did not cause any weight loss in mice (Supplementary Fig. 8e), morphological changes in liver and lung tissues assessed by H\&E staining (Supplementary Fig. 8f), or alterations in the survival of NPCs residing in the subventricular zone (SVZ) of mouse brains (Supplementary Fig. 8g). These data suggest that RocA treatment selectively targets GSCs and inhibits the growth of orthotopic GBM xenografts but has no severe toxicity on normal tissues.

We then assessed whether targeting PHB by RocA synergizes with IR in GBM treatment. Our results showed that combined RocA and IR treatment dramatically induced peroxide levels (Fig. 7a and Supplementary Fig. 9a) and increased cell apoptosis in GSCs (Fig. 7b, c and Supplementary Fig. 9b,c). Furthermore, RocA treatment sensitized GSCs to IR treatment in vitro (Fig. 7d).
Importantly, in two established orthotopic GBM xenografts, the combination of RocA and IR treatment yielded the greatest efficacy in suppressing tumor growth and extending overall survival relative to RocA or IR treatment alone (Fig. $7 \mathrm{e}-\mathrm{g}$ ). The therapeutic efficacy of the combination treatment was further validated in GBM patient-derived xenografts (PDXs) (Fig. 7h, i). Meanwhile, TUNEL staining showed that the combination treatment resulted in a remarkable increase in cell death in tumors, whereas RocA or IR alone increased tumor cell apoptosis, validating the synergistic effect of RocA and IR treatment on inhibiting GBM growth (Supplementary Fig. 9d). To further evaluate the potential therapeutic value of RocA in GBM treatment, we compared the tumor inhibitory effects of RocA and TMZ. Our data indicated that RocA was superior to TMZ treatment in inhibiting GSC growth in vitro (Supplementary Figs. 7c, 9e). Moreover, the combined RocA and TMZ treatment 


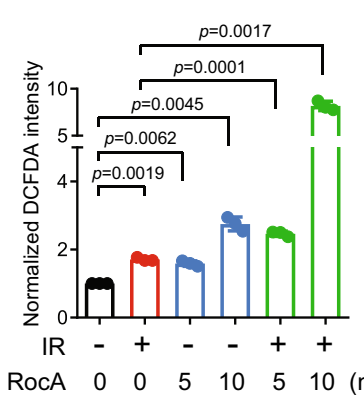

b

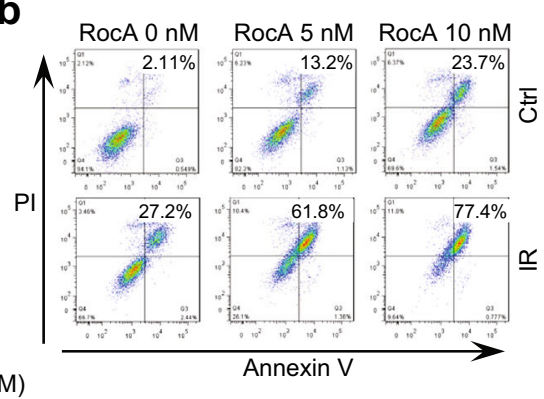

C

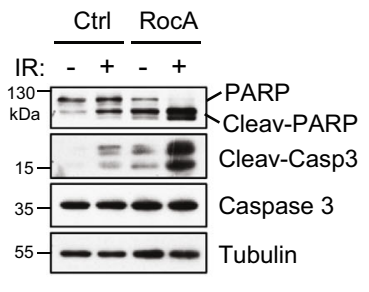

d

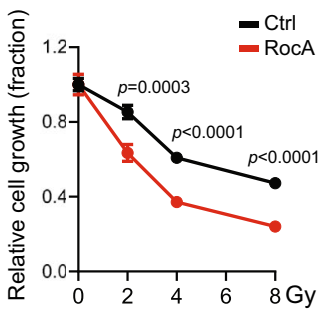

e

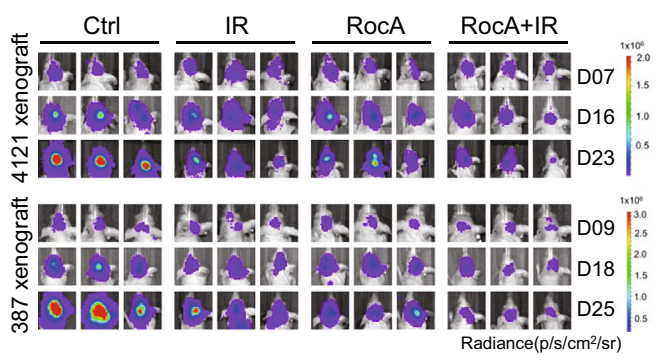

f

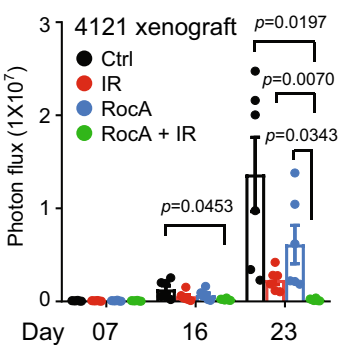

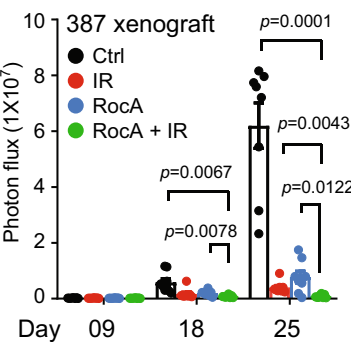

g

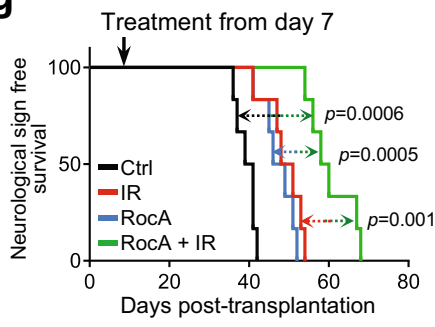

h

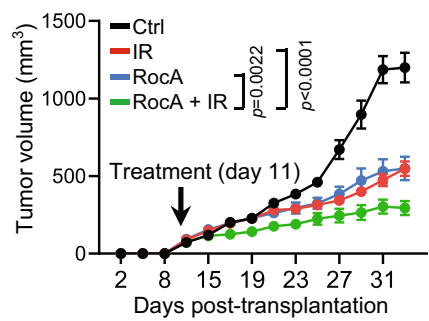

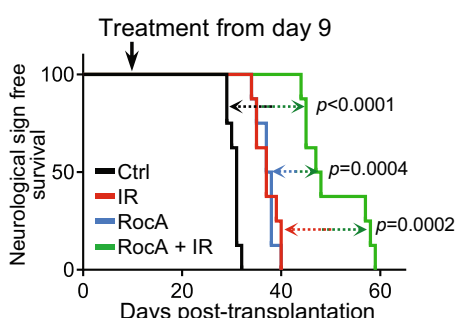

i

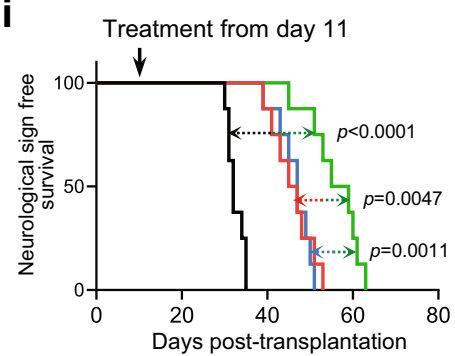

Fig. 7 Pharmacological targeting PHB increases sensitiveness of GSCs to IR. a The peroxide levels, as indicated by DCFDA fluorescence, were measured by flow cytometry in 4121 GSCs with indicated treatment for $48 \mathrm{~h}$. IR, $3 \mathrm{~Gy}$ (mean $\pm \mathrm{SD}, n=3$, biologically independent experiments, Welch's two-sided $t$ test). b Cell apoptosis was measured by flow cytometry in 4121 GSCs with indicated treatments for $48 \mathrm{~h}$. IR, $3 \mathrm{~Gy}$. c IB showing levels of cleaved-PARP, cleaved-caspaspe3, and caspase3 in 4121 GSCs with indicated treatments for 48 h. IR, 3 Gy. d Cell growth of 4121 GSCs treated with indicated doses of IR for $48 \mathrm{~h}$ in the absence or presence of RocA $(5 \mathrm{nM})$. Data were normalized to the untreated cells of each group (mean $\pm S D, n=4$, biologically independent experiments, unpaired two-sided Student's t-test). e-g Nude mice (nu/nu) intracranially implanted with GSCs (Luciferase) were randomly grouped (4121 GSCs, $n=6 ; 387 \mathrm{GSCs}, n=8$, for each group) and treated with control, IR (3 Gy, once a week, three times), RocA ( $2.5 \mathrm{mg} / \mathrm{kg}$, every 3 days, six times), or the combined treatment from day 7 (4121 GSC xenografts) or day 9 (387 GSC xenografts). GBM xenografts were tracked by bioluminescence and the representative images are shown (e). Bioluminescent quantifications of tumor growth are shown (f) (mean \pm SEM, Welch's two-sided $t$-test).

Kaplan-Meier survival plots of mice are shown (g, 4121 GSCs, left; 387 GSCs, right) (Log-rank Mantel-Cox test). h, i NOD/SCID mice were subcutaneously implanted with GBM PDX tumors. Mice were randomly grouped ( $n=8$ for each group) at day 11 and treated with control, IR ( $3 \mathrm{~Gy}$, once a week, 4 times), RocA ( $2.5 \mathrm{mg} / \mathrm{kg}$, every 3 days, eight times in total) or the combined treatment. Tumor volume was measured (h) (mean \pm SEM, Two-way ANOVA). Kaplan-Meier survival plot of mice is shown (i). (Log-rank Mantel-Cox test). Source data are provided as Source Data file.

did not show a dramatic increase in GSC apoptosis in vitro (Supplementary Fig. 9f), or a strong synergistic therapeutic effect on GBM growth in vivo (Supplementary Fig. 9g). Collectively, these results demonstrate that targeting GSCs by RocA effectively suppresses GSC-derived tumor growth and significantly improves the efficacy of radiotherapy for GBM.

\section{Discussion}

ROS play crucial roles in stem cell maintenance ${ }^{42,54}$. Our results revealed that GSCs maintain low mitochondrial ROS content that is similar to NPCs, when compared to levels in NSTCs. This raises an important question regarding how to eliminate GSCs by targeting the ROS-mediated mechanisms without affecting normal stem cells. In this study, we revealed that PHB is a novel regulator that specifically controls ROS levels in GSCs. PHB is highly expressed in GSCs relative to NPCs, NHA, and NSTCs. Although disruption of PHB strongly increases ROS accumulation and compromises GSC self-renewal both in vitro and in vivo, abrogation of PHB has a weak inhibitory effect on NPCs or NHA growth, highlighting the unique role of $\mathrm{PHB}$ in ROS regulation in GSCs.

$\mathrm{PHB}$ is an evolutionarily conserved protein that has been implicated in diverse cellular processes, including cell proliferation, replicative senescence, and mitochondrial biogenesis ${ }^{24,25}$. However, how PHB act at the molecular level is not yet fully understood. Studies indicate that PHB may be involved in the regulation of complex I of mitochondrial electron transport chain and in mitochondrial respiration $25,44,55$. However, our data indicated that the respiration and activity of complex I in mitochondria are not significantly impaired by PHB deletion in GSCs, which is consistent with other previous findings ${ }^{56}$, suggesting that the roles of $\mathrm{PHB}$ may be cell-type dependent. Our results demonstrated that PHB associates with and stabilizes PRDX3, a peroxiredoxin protein that scavenges peroxides and protects cells from oxidative stress ${ }^{22}$. The function of PHB is likely mediated by PRDX3, as overexpressing PRDX3 or scavenging ROS by NAC rescues the induction of ROS and the inhibition of self-renewal caused by PHB deletion in GSCs. We further demonstrated that 
PHB protects PRDX3 from ubiquitin-proteasome-dependent degradation; however, the detailed molecular mechanisms require further elucidation.

GBM is the most common primary brain malignancy in adults with no effective treatment. Standard non-surgical treatment, including radio-chemotherapy, produces only modest benefits partially due to the resistance of GSCs ${ }^{4,7,39}$. Understanding the underlying mechanisms of resistance may provide new strategies to improve GBM treatment. We demonstrated that low levels of ROS mediated by PHB protect GSCs from IR-induced cell killing and promote GBM therapeutic resistance. Targeting PHB by genetic deletion or pharmacological inhibition effectively suppresses tumor growth, overcomes the resistance of GSCs to radiotherapy, and thus extends animal survival in preclinical GBM models. Meanwhile, the percentage of PHB + cells is increased in high-grade gliomas and positively correlated with the recurrence and poor prognosis of glioma patients, reinforcing that PHB may be an attractive target for GBM therapy.

Rocaglamides are natural products that have been observed to possess antitumor activities in various tumor cell lines ${ }^{57}$. Although studies suggest that the primary effect of RocA may be inhibition of protein synthesis ${ }^{50}$, its direct molecular targets remain largely unknown. Recently, a study has shown that RocA directly binds to $\mathrm{PHB}$ and blocks the membrane associations between PHB and c-Raf ${ }^{48}$. In our study, we found that RocA binds to PHB and decreases the interaction between PHB and PRDX3. Notably, a very low dose of RocA $(<10 \mathrm{nM})$ recapitulates the findings observed with PHB deletion, including the induction of mitochondrial ROS and the inhibition of cell growth in GSCs with limited toxicity in NHA or NPCs. Meanwhile, the overexpression of PRDX3 largely attenuates the induction of ROS by RocA and blocks its inhibitory effects in GSCs. These findings suggest that the effects of RocA are likely mediated through the inhibition of PHB-PRDX3 signaling in GSCs. Our data revealed that low doses of RocA result in no obvious impact on the c-Raf/ ERK, NF- $\mathrm{kB}$, or eIF4A signaling pathways ${ }^{48,50,51}$, suggesting that the action mode of RocA may be cell-type dependent and associated with the subcellular localization of PHB. Most importantly, RocA treatment significantly inhibits GBM tumor growth and provides survival benefits without any detectable signs of sickness or morbidity in tumor-bearing mice. Combination treatment with RocA and radiotherapy represents a strong synergistic anti-tumor effect in established orthotopic GBM xenografts and GBM PDXs, supporting the potential of RocA for clinical application in GBM treatment both as a monotherapy and as an adjuvant therapy with radiation.

\section{Methods}

Cells, tissues, and cell culture. GSCs were isolated from GBM surgical specimens or xenografts and were functionally characterized ${ }^{32-35}$. Briefly, cells were isolated from primary GBM specimens or patient-derived GBM xenografts according to the Papain Dissociation System (Worthington Biochemical) manufacturer's instructions and recovered in the stem cell medium (Neurobasal-A medium with B27 supplement, $10 \mathrm{ng} / \mathrm{ml} \mathrm{EGF,} 10 \mathrm{ng} / \mathrm{ml} \beta$ FGF, $1 \%$ penicillin/streptomycin, $1 \mathrm{mM}$ sodium pyruvate, and $2 \mathrm{mM} \mathrm{L}$-glutamine) overnight in a humidified incubator with $5 \% \mathrm{CO}_{2}$. GSCs were then sorted by magnetic cell sorting using the surface marker CD133 (Miltenyi Biotec.) and cultured in stem cell medium as described above and assayed for expression of stem cell markers including SOX2, Olig2, Nestin, and absence of GFAP expression. A series of functional assays were then applied to validate the cancer stem cell phenotypes of the isolated GSCs, including the sphereforming ability (in vitro limiting dilution assay), multipotent differentiation ( $10 \%$ fetal bovine serum induction of multi-lineage differentiation in vitro), and tumorinitiation in immunocompromised mice in our previous studies ${ }^{32,33}$. Specifically, 4121 GSCs were derived from a recurrent GBM patient (53-year old, male); 387 GSCs were derived from a primary GBM patient (76-year old, female); 3691 GSCs were derived from a primary GBM patient (59-year old, female); 456 GSCs were derived from a primary GBM patient (8-year old, female); 3832 GSCs were derived from a primary GBM patient (75-year old, female).

NSTCs (CD133-) were isolated from patient-derived GBM xenografts and cultured in Dulbecco's modified Eagle's medium (DMEM) supplemented with 10\%
FBS to maintain differentiation status. Low $(<5)$ passage cells were used for experiments to prevent cellular drift. For cell differentiation assay, GSCs were induced for differentiation by the withdrawal of growth factors and by the addition of 10\% FBS in DMEM. On the indicated day, cells were harvested for IB analysis.

Three human NPC lines (hNP1, 15167, 17231, derived from fetal brains, Lonza) were cultured and maintained in the stem cell medium described above according to the vendor's instruction. NHA (Beina Chuanglian Biotechnology Institute) was maintained in glucose-free DMEM supplemented with $5 \mathrm{mM}$ glucose and $10 \%$ FBS.

GBM surgical specimens were collected with the approval of the PLA General Hospital. Informed consent was obtained for all subjects. Specifically, G\#7 was from a GBM patient (56-year old, male); G\#19 was from a GBM patient; G\#27 from a GBM patient (56-year old, male); G\#28 from a GBM patient (73-year old, female); G\#29 from an Astrocytoma patient (Grade III, 53-year old, male); G\#32 from a GBM patient. The glioma tissue microarray was purchased from Shanghai Outdo Biotechnology Company, Ltd (HBraG171Su01). Histologic diagnosis of the tissue microarray cores was reviewed by at least two individuals, one of whom is a pathologist. 11 specimens were removed because of tissue incompletion or mismatching with the pathological diagnosis. The tissue microarray included tumors from 94 patients with grade I-II astrocytoma, 35 females, 59 males, ages 3-80 years old, median 41 years; 46 patients with grade III astrocytoma, 19 females, 27 males, ages 17-68 years old, median 43 years; 20 patients with GBM, 3 females, 17 males, ages 9-78 years old, median 46 years. The patients' information of the tissue microarray is listed in Supplementary Table 1.

Orthotopic mouse xenografts. All animal experiments were performed in accordance with the NIH guide for the care and use of laboratory animals and with the approval of the Institutional Animal Care and Use Committee of National Center of Biomedical Analysis. Mice used in our studies were 4-week-old female NU/NU nude mice purchased from Beijing Vital River Laboratory Animal Technology. Mice had not undergone prior treatment or procedures. Animal care was monitored daily by certified veterinary staff and laboratory personnel. Five mice were housed per cage, with a $14 \mathrm{~h}$ light $/ 10 \mathrm{~h}$ dark cycle, and were provided with food and water. Animals were monitored until neurological signs were observed, at which point they were sacrificed by inhalation of carbon dioxide followed by cervical dislocation. All surgical procedures were performed under anesthesia by intraperitoneal injection of a ketamine and xylazine cocktail. Tissues were removed following euthanasia and were fixed in $4 \%$ paraformaldehyde (PFA, SigmaAldrich) at $4{ }^{\circ} \mathrm{C}$ for $24 \mathrm{~h}$, stored in $30 \%$ sucrose solution at $4{ }^{\circ} \mathrm{C}$ for $48 \mathrm{~h}$, embedded in OCT at $-80^{\circ} \mathrm{C}$ overnight, and cryosection thickness of 7 microns. Methods describing the establishment of mouse orthotopic xenograft are described below.

Immunofluorescence staining, immunohistochemistry, and IB. For the Immunofluorescent staining experiments, the cultured cells, tissue cryosections, or human surgical specimens were fixed in $4 \%$ PFA for $15 \mathrm{~min}$, and were then permeabilized in PBS containing 0.3\% Triton X-100 (Bio-Rad Laboratories) for 15 min. Samples were blocked with $5 \%$ albumin from bovine serum with $0.3 \%$ Triton X-100 in PBS for $60 \mathrm{~min}$ at room temperature and then incubated with primary antibodies overnight at $4{ }^{\circ} \mathrm{C}$ followed by the appropriate secondary fluorescently labeled antibodies for one hour at room temperature. Nuclei were counterstained with Hoechst (Invitrogen). Images were acquired using a laser confocal microscope (Zeiss, LSM880) with Zen 2.1 SP2 software, and were processed using Image J software 1.8.0.

For the immunohistochemical staining experiments, a tissue microarray of deidentified formalin-fixed, paraffin-embedded glioma specimens was immunostained with PHB antibody. Secondary antibody was labeled with polymer-HRP (horseradish peroxidase) anti-rabbit as appropriate. Staining was visualized using 3,30-diaminobenzidine (DAB) chromogen (Zhongshan Golden Bridge). Presence or absence of PHB staining was scored by at least two individuals, one of whom is a pathologist, and consensus scores are reported. Briefly, the staining intensity of PHB has assessed both the intensity of the staining and the percentage of positively stained cells. For the intensity, a score of $0-3$ (corresponding to negative, weak, moderate, or strong staining) was recorded and the percentage of positively stained cells at each intensity was estimated.

Immunoblotting was carried out following standard methods ${ }^{32}$. Briefly, cells were lysed in lysis buffer (Tris-HCl $20 \mathrm{mM} \mathrm{PH} \mathrm{7.4,0.5 \%} \mathrm{NP-40,} 250 \mathrm{mM} \mathrm{NaCl}$, $3 \mathrm{mM}$ EGTA, $3 \mathrm{mM}$ EDTA) supplemented with protease inhibitors (Roche) and incubated on ice for $30 \mathrm{~min}$. The Bradford assay (Bio-Rad Laboratories) was utilized for the determination of protein concentration. Equal amounts of protein were mixed with reducing Laemmli loading buffer, boiled for $10 \mathrm{~min}$, and resolved by SDS-PAGE, then transferred onto PVDF membranes (Millipore, Billerica, MA). Blots were incubated with primary antibodies overnight at $4^{\circ} \mathrm{C}$ followed by HRPconjugated species-specific antibodies (Jackson Immuno Research, 1:5000) at room temperature for one hour.

The following antibodies were used: PHB (Abcam for IB 1:5000; for IF, 1:200; for IHC 1:100), SOX2 (Millipore for IB, 1:1000; Santa Cruz for IF, 1:200), Olig2 (Santa Cruz for IB, 1:1000; R\&D for IF, 1:200), GFAP (Dako for IB:1000), PRDX3 (Novus for IB, 1:5000; for IF, 1:200), 8-OHdG (Abcam for IF, 1:400), TOM20 (Santa Cruz for IF, 1:400), TIM23 (Santa Cruz for IF,1:200), Tubulin (Sigma for IB, 1:5000), Caspase3 (Proteintech for IB, 1:1000), Cleaved-Caspase3 (Cell Signaling 
for IB, 1:1000; for IF, 1:100), PARP (Cell Signaling for IB, 1:1000), Flag (Sigma for IB, 1:2000), PRDX1 (Proteintech for IB, 1:1000), PRDX6 (Proteintech for IB, 1:1000), GPX1 (Abcam for IB, 1:1000), Mn-SOD (Proteintech for IB, 1:1000), PHB2 (Cell Signaling for IB,1:1000), $\beta$-Actin (Santa Cruz for IB, 1:1000), Ubiquitin (MBL for IB, 1:1000), Ubiquitin, Lys48-specific (Millipore for IB, 1:1000), p65 (Santa for IB, 1:500), phospho-p65 (Cell Signaling for IB, 1:1000), c-Raf (Cell Signaling for IB, 1:1000), Phospho-c-Raf (Cell Signaling for IB,1:1000), ERK (Cell Signaling for IB, 1:500), Phospho-p44/42 MAPK (Erk1/2) (Cell Signaling for IB, 1:500), eIF4A1 (Cell Signaling for IB, 1:1000), Notch1 (Cell Signaling for IB, 1:1000), c-Myc (Cell Signaling for IB, 1:1000), Bcl-2 (Santa Cruz for IB, 1:500), Ezh2 (Cell Signaling for IB, 1:1000), Cu-ZnSOD (Proteintech for IB, 1:1000), $\gamma \mathrm{H} 2 \mathrm{AX}$ (Millipore for IB, 1:1000). Secondary antibody labeled with polymer-HRP anti-rabbit (Jackson for IB, 111-035-003, Lot:147832, 1:5000), secondary antibody labeled with polymer-HRP anti-mouse (Jackson for IB,115-035-003, Lot:148148, 1:5000). Alexa Fluor 488 donkey anti-rabbit IgG (Thermo for IF, A21206, Lot:1927937, 1:400), Alexa Fluor 488 donkey anti-mouse IgG (Thermo for IF, A21202, Lot:1915874, 1:400), Alexa Fluor 555 donkey anti-mouse IgG (Thermo for IF, A31570, Lot:1905844, 1:400), and Alexa Fluor 555 donkey anti-goat IgG (Thermo for IF, A21432, Lot:1932497, 1:400).

DNA constructs and lentiviral transfection. Human Flag-PHB and Flag-PRDX3 were generated by PCR and were cloned into the pCDH-MCS-T2A-Puro-MSCV lentiviral vectors (System Biosciences). The truncated mutant forms of PHB (1-90aa, 1-173aa, 41-272aa, 174-272aa) were cloned into the pCDH-MCS-T2APuro-MSCV vectors. The truncated mutant forms of PRDX3 (1-221aa, 63-221aa, 63-256aa) were cloned into the pGEX-4T-1 vector (Adgene, \#27-4580-01). Clones expressing two non-overlapping sgRNAs directed against human $P H B$ or a nontargeting control sgRNA that has no targets in the human genome were cloned into the LentiGuide-puro vectors (Addgene); Clones expressing PHB DOX-induced shRNAs were cloned into the Tet-pLKO-puro vectors (Addgene); Clones expressing PHB, PRDX3 or EIF4A shRNAs were cloned into the pLKO.1 TRC vectors (Addgene). The sgRNAs and shRNAs used in this study are listed in Supplementary Table 2.

HEK293 cells were used to generate lentiviral particles through co-transfection of the packaging vectors pSPAX2 and pVSVG (Addgene) using a standard calcium phosphate transfection method. For rescue experiments, GSCs stably expressing Flag-PRDX3 were transduced with PHB shRNAs lentiviral constructs. After recovering for $48 \mathrm{~h}$, cells were selected by puromycin $(2 \mu \mathrm{g} / \mathrm{ml})$.

CRISPR-Cas9 gene knockout. The CRISPR design tool from the Broad Institute (https://portals.broadinstitute.org/gpp/public/analysis-tools/sgrna-design) was used to design PHB sgRNAs. Oligonucleotides were annealed and cloned into LentiGuidepuro (Addgene) plasmid. For knockout studies, GSCs were transduced with LentiCas9-blast (Addgene) lentiviral construct and selected by blasticidin $(10 \mu \mathrm{g} / \mathrm{ml})$ to obtain GSCs stably expressing Cas9. After that, the GSCs-Cas9 were transduced with PHB sgRNAs lentiviral constructs and selected by puromycin $(2 \mu \mathrm{g} / \mathrm{ml})$. Cell pools were harvested to confirm the knockout efficiency of PHB by IB.

Inducible KD. GSCs expressing PHB DOX-induced shRNAs were cultured with $100 \mathrm{ng} / \mathrm{ml}$ DOX for 4 days before cell lysates were collected to confirm the KD efficiency of PHB. For in vivo inducible KD, GSCs expressing DOX-induced PHB shRNAs were intracranially transplanted into nude mice, supplied with water containing $2 \mathrm{mg} / \mathrm{ml}$ DOX. The growth of orthotopic GBM tumors was monitored by bioluminescence imaging using the Calibar IVIS ${ }^{\oplus}$ Spectrum (PerkinElmer) in vivo imaging system.

Cell viability assays. 2000 cells were seeded in 96 -well plates with $200 \mu$ culture medium. Cell viability was measured using CellTiter-Glo (Promega, Madison) after the indicated number of days. All data were performed in triplicate.

Immunoprecipitation and pull-down assay. Cells were collected and lysed in NP40 lysis buffer (Boster biological technology, AR0107) supplemented with protease inhibitors, incubated on ice for $30 \mathrm{~min}$, and followed by centrifugation at $15,000 \times \mathrm{g}$ for $15 \mathrm{~min}$ at $4{ }^{\circ} \mathrm{C}$. The supernatant was subjected to immunoprecipitation with the primary antibody ( $5 \mu \mathrm{g}$ of antibody, or normal rabbit or mouse IgG) overnight at $4^{\circ} \mathrm{C}$. The precipitants were extensively washed six times with lysis buffer, boiled with SDS loading buffer and subjected to SDS-PAGE. For the mapping experiments, HEK293 cells were co-transfected with HA-PRDX3 and the full-length or truncated mutants of Flag-PHB for $48 \mathrm{~h}$, and then were treated as described above.

RocA (MCE, HY-19356) was conjugated with cyanogen bromide (CNBr)activated Sepharose 4B (Sangon Biotech, C500099-0010) in coupling buffer $(0.1 \mathrm{M}$ $\mathrm{NaHCO}_{3}, 0.5 \mathrm{M} \mathrm{NaCl}, \mathrm{pH} 8.3$ ) at $4{ }^{\circ} \mathrm{C}$ overnight. Cells were collected and lysed in RIPA lysis buffer ( $20 \mathrm{mM}$ Tris-HCl, pH 7.5; 0.5\% NP-40; $150 \mathrm{mM} \mathrm{NaCl} ; 10 \mathrm{mM}$ EDTA; $1 \%$ Triton X-100; $1 \%$ deoxycholate and $\mathrm{ddH}_{2} \mathrm{O}$ ) containing complete protease inhibitor cocktail (Roche, 04693132001 ) for $30 \mathrm{~min}$, followed by centrifugation at $21,000 \times g$ for $20 \mathrm{~min}$ at $4{ }^{\circ} \mathrm{C}$. The supernatants were incubated with RocA-conjugated Sepharose $4 \mathrm{~B}$ at $4{ }^{\circ} \mathrm{C}$ overnight. The precipitants were extensively washed six times with lysis buffer, boiled with SDS loading buffer and subjected to SDS-PAGE.
GST-pull down assay. GST-vector and GST-PRDX3 were expressed in BL21 Competent E. coli, purified and incubated with Glutathione Sepharose 4B beads (GE Healthcare, 17-0756-01) according to the manufacturer's protocol (Amersham Biosciences). For binding assay, Flag-PHB was transfected into HEK293 cells and then was lysed with NP-40 lysis buffer. The cell lysate supernatant was subjected to GST-beads coupled PRDX3 overnight at $4{ }^{\circ} \mathrm{C}$ in NP-40 lysis buffer. The precipitants were extensively washed five times with lysis buffer, boiled with SDSloading buffer and subjected to SDS-PAGE.

RNA isolation and real-time PCR. Cell pellets were collected and the total RNA was extracted using RNeasy kit (QIAGEN), then reversely transcribed to cDNA with PrimeScript ${ }^{\mathrm{TM}}$ RT Master Mix (Takara Bio Inc.) according to the manufacturer's instructions. Real-time PCR was performed with SYBR Green Master Mix (Applied Biosystems) on a cycler (Applied Biosystems). GAPDH or Actin was used for normalization. For miRNA-27a detection, all the Bulge-Loop RT primers for both miRNA-27a and U6 were purchased from RiboBio Co., Ltd. (Guangzhou, China). The relative miRNA levels were normalized to small nuclear RNA U6. The primer pairs used to detect the mRNA and miRNA levels are listed in Supplementary Table 3.

Oligonucleotides and cell transfection. The Homo sapiens (hsa) miRNA-27a mimics (miR-27a sense oligonucleotides), miR-27a inhibitor (miR-27a anti-sense oligonucleotides), and the controls were purchased from RiboBio Co, Ltd. (Guangzhou, China). The mimic and inhibitor control were scrambled oligonucleotide that not produce any identifiable effects on known miRNA function. Cells were transfected with the oligonucleotide using Lipofectamine 3000 (Thermo Fisher Scientific, L3000015) according to the manufacturer's instruction.

3'-UTR-luciferase reporter assay. The wild type and mutant $3^{\prime}$-UTR regions of PHB containing predicted miR-27a target site were synthesized (Tsingke Biotechnology Co., Ltd.) and were cloned into the pGL3 vector (Promega, E1741) immediately downstream of the stop codon of the luciferase gene. Either the wildtype or mutant $P H B 3^{\prime}$-UTR-luciferase was co-transfected with miR-27a mimic/ inhibitor in GSCs using Lipofectamine. PRL-TK internal control vector (Promega, E2241) was co-transfected as the endogenous control for luciferase activity. After $48 \mathrm{~h}$, luciferase activity was measured using Dual-Luciferase Reporter Assay (Promega, E1910). The luciferase activity was normalized to PRL-TK activity.

Determination content of GSH. GSCs were treated with or without $50 \mu \mathrm{M}$ L-BSO (MedChemExpress, HY-106376A) for $48 \mathrm{~h}$ before collection. GSH detection was performed according to the manufacturer's instructions (Beyotime Biotechnology, S0053). Briefly, cells were washed with $1 \times$ PBS and collected, re-suspended with three times the volume of protein removal reagent $\mathrm{M}$ solution. Cell samples were subjected to two rapid freeze-thaw cycles using liquid nitrogen and $37^{\circ} \mathrm{C}$ water bath. Corresponding detection reagents were added to an appropriate amount of cell samples. After $25 \mathrm{~min}$, GSH was detected by a microplate analyzer at an absorbance of $412 \mathrm{~nm}$. Then GSH content was calculated according to the standard curve.

Determination activity of catalase. GSCs were treated with or without $50 \mu \mathrm{M}$ EGCG (MedChemExpress, HY-13653) for $24 \mathrm{~h}$ before collection. The determination activity of catalase was performed according to the manufacturer's instructions (Beyotime Biotechnology, S0051). Briefly, cells were lysed in lysis buffer (Tris-HCl 20 mM PH 7.4, 0.5\% NP-40, $250 \mathrm{mM} \mathrm{NaCl}, 3$ mM EGTA, 3 mM EDTA) supplemented with protease inhibitors (Roche) and incubated on ice for $30 \mathrm{~min}$. Cell lysis samples were mixed with the corresponding test solution. After $20 \mathrm{~min}$, catalase activity was detected by a microplate analyzer at an absorbance of $240 \mathrm{~nm}$.

In vitro limiting dilution assay. For in vitro LDA, decreasing numbers of cells per well $(200,150,100,50$, and 20$)$ were plated in 96-well plates with 12 replicates for each cell number. Ten days after plating, the presence and number of neurospheres in each well were quantified. Extremely limiting dilution analysis was performed using software available at http://bioinf.wehi.edu.au/software/elda.

Establishment of GSC-derived intracranial GBM xenografts and combination treatment. Orthotopic GBM xenografts were established through intracranial transplantation of GSCs ${ }^{32,33}$. Briefly, mice used in the studies were 4 -week old, female, nu/nu nude mice. GSCs $\left(5 \times 10^{4}\right.$, or indicated number $)$ with indicated treatment were implanted into the right frontal lobe of mice. Mice in each group were treated with one of the following: vehicle control, DOX $(1 \mathrm{mg} / \mathrm{ml}$, daily in water) or RocA ( $2.5 \mathrm{mg} / \mathrm{kg}$, i.p., every 3 days, $6-8$ times in total), Irradiation $(3 \mathrm{~Gy}$, once a week, four times in total)/TMZ $(60 \mathrm{mg} / \mathrm{kg}$, i.p., every 3 days, six times in total), or the combination of DOX/RocA and IR/TMZ starting on the indicated days after tumor implantation. IR was performed with a $\gamma$-ray irradiator (Co-60). Xenograft growth was monitored by bioluminescent imaging using the Calibar IVIS $^{\circ}$ Spectrum (PerkinElmer) in vivo-imaging system every week. 


\section{Measurements of mitochondrial ATP and oxygen consumption. For mito-} chondrial ATP and oxygen consumption measurement, GSCs were seeded in the $\mathrm{XF}$ cell culture microplate (Agilent), intact cellular oxygen consumption was performed using a Seahorse XF96 analyzer. Cells were sequentially challenged with oligomycin $(1 \mu \mathrm{M})$, carbonyl cyanide 4-(trifluoromethoxy) phenylhydrazone (FCCP) $(1 \mu \mathrm{M})$, and rotenone plus antimycin $\mathrm{A}(1 \mu \mathrm{M})$.

Intracellular and mitochondrial ROS quantification. Cells were harvested and trypsinized as a single cell, washed with PBS, re-suspended in $10 \mu \mathrm{M} \mathrm{CM}$ H2DCFDA (Thermo Fisher Scientific, c6827) in PBS and stained for $15 \mathrm{~min}$ at $37^{\circ} \mathrm{C}$ in the incubator. After staining, cells were washed twice with PBS and then re-suspended in PBS. H2DCFDA fluoresce signaling was detected by flow cytometer (BD Aria III) at $488 \mathrm{~nm}$ channel, and totally 10,000 cells were analyzed per sample. Data analysis was performed with FlowJo 7.6 software.

Apoptosis assay. After indicated treatments, cells were harvested and trypsinized as single cell. Cells were re-suspended in $100 \mu \mathrm{l}$ AnnexinV-FITC/PI buffer (Promega) and incubated for $15 \mathrm{~min}$ at room temperature in dark. The rate of apoptosis was measured by a flow cytometer (BD Aria III). Totally 10,000 cells were analyzed per sample. Data analysis was performed with FlowJo 7.6 software.

Ubiquitination. GSCs were treated with $10 \mu \mathrm{M}$ MG132 for $12 \mathrm{~h}$ before collection. Cells were lysed in NP-40 lysis buffer (Boster biological technology, AR0107) supplemented with protease inhibitors (Roche) and N-Ethylmaleimide (Sigma, E3876), incubated on ice for $30 \mathrm{~min}$, and followed by centrifugation at $15,000 \times g$ for $15 \mathrm{~min}$ at $4{ }^{\circ} \mathrm{C}$. The supernatant was subjected to immunoprecipitation with anti-Flag M2 affinity gel (Sigma, A2220) or protein G sepharose (GE Healthcare, 17-0618-01). The immunoprecipitants were detected by immunoblotting with antiubiquitin antibody, anti-ubiquitin Lys48-specific antibody, or anti-Flag antibody to detect ubiquitination of PRDX3.

RNA sequencing. Total RNA was isolated from cells using the RNeasy Mini Kit (QIAGEN). Strand-specific cDNA libraries were generated using the Illumina TruSeq Stranded Total RNA Library Prep Kit with Ribo-Zero Gold. cDNA quality was determined using the Agilent high-sensitivity DNA kit on an Agilent 2100 BioAnalyzer (Agilent Technologies). Paired-end $125 \mathrm{bp}$ reads were generated on an Illumina HiSeq 2500 instrument at the Oebiotech.corp. Reads were aligned to the GRCh38.p7 genome using TopHat v2.1.1 with the library type option set to first strand. Fragments per kilobase per millions (FPKMs) of known genes were calculated using eXpress v1.5.1.

PDX models. PDXs were established through subcutaneous transplantation of GBM patient cells into NOD/SCID mice. Mice used in the studies were 4-week old, female. After mass formation, mice were randomly divided into four groups, treated with one of the followings: vehicle control, RocA $(2.5 \mathrm{mg} / \mathrm{kg}$, i.p., every 3 days, eight times), IR (3 Gy, once a week, four times), or the combination of RocA and IR starting on the indicated days after tumor implantation. IR was performed with a $\gamma$-ray irradiator (Co-60). Tumor size was evaluated every 2 days by caliper measurements and the approximate volume of the mass was calculated using the formula [(small diameter $)^{2} \times($ large diameter $\left.) \times 0.5\right]$. Once tumor volume exceeded $1500 \mathrm{~mm}^{3}$, mice were sacrificed.

Liquid chromatography-tandem mass spectrometry analysis of Rocaglamide. The smashed mouse brains and extracted serums were added with $80 \%$ mixture of methanol and acetonitrile $(1: 1)$, pre-chilled at $-80^{\circ} \mathrm{C}$ and incubated at $-80^{\circ} \mathrm{C}$ for $1 \mathrm{~h}$. Then, the samples were centrifuged at $18,000 \times g$ for $10 \mathrm{~min}$ and the supernatant was dried with SpeedVac. The pellets were rediscounted with $100 \mu \mathrm{l}$ of $50 \%$ methanol solution and centrifuged at $18,000 \times g$ for $10 \mathrm{~min}$ at $4{ }^{\circ} \mathrm{C}$ for supernatants collection. Extracted samples and standards were analyzed by Triple Quad 6500 mass spectrometer (SCIEX) coupled with the UltiMate 3000 HPLC system (Thermo Fisher Scientific). The LC separation was performed on an ACQUITY UPLC HSS T3 column $(100 \mathrm{~mm} \times 2.1 \mathrm{~mm})$ (WATERS). For Rocaglamide multiple reaction monitoring (MRM) detection, 506.0/352.1 transition was chosen in positive ion mode. Peak integration and statistical analyses were performed using MultiquantTM 2.1 software (SCIEX).

Microarray analysis from GEO database. Microarray data of GEO (http://www. ncbi.nlm.nih.gov/geo) GSE86237 and GSE54791, were enrolled in the study. GSE86237 profile included microarray data from 19 panels of GSCs and 7 panels of BTCs both isolated from primary GBM samples ${ }^{36}$. GSE54791 database contained gene-profiling data from three pairs of GSCs and NSTCs ${ }^{37}$.

Statistics and reproducibility. All grouped data are presented as mean \pm SD or SEM from studies performed at least in triplicate unless otherwise specified. For bar graphs, the unpaired two-sided Student's $t$-test or Welch's two-sided $t$-test was used for the comparison between unpaired two groups. Two-way ANOVA was applied for multi-group data comparison. A probability value $<0.05$ was considered significant. For the survival analysis, Kaplan-Meier survival curves were analyzed by using Log-rank Mantel-Cox test comparing the different patient or mouse groups. $\mathrm{IC}_{50}$ of RocA was calculated using nonlinear regression analyses based on dose-response curves. For all figures presented in box-and-whisker format, the center line represents the median and the lower and upper limits of the box represent the 25th and 75th percentiles. The maximum and minimum are connected to the center box through the vertical lines (whiskers). GraphPad Prism Software 8.0 (GraphPad Software, Inc.) and Microsoft Office Excel (office 2013) were used for all statistical analyses.

Results in Figs. 1a-c, g, h, 2a, b, 4a-d, f, g (left and right panels), h, 5i, 6a-c; Supplementary Figs. 1c, g, h, 2h, 3g, 4a, b, 5c, g, 5h, i (right panels), k, p, 6f, 6i, j, $7 \mathrm{~g}-\mathrm{j}, 9 \mathrm{c}$. are representative data of three independent repeats. And there were similar results in three independent repeats.

Reporting summary. Further information on research design is available in the Nature Research Reporting Summary linked to this article.

\section{Data availability}

The microarray data referenced during the study are available from the Gene Expression Omnibus under accession numbers GSE86237 and GSE54791. The RNA sequencing data for control GSCs and PHB KO GSCs have been deposited in the public database under the accession code HRA000843. The expression of PHB and the pathological characteristics of human glioma patients of the tissue microarray is provided in Supplementary Table 1. The sgRNAs and shRNAs used in this study are provided in Supplementary Table 2. The primer sequences used for Q-PCR are provided in Supplementary Table 3. Source data are provided with this paper. The remaining data are available within the Article, Supplementary Information and Source Data. Source data are provided with this paper.

Received: 9 November 2020; Accepted: 2 June 2021; Published online: 17 June 2021

\section{References}

1. Stupp, R. et al. Effects of radiotherapy with concomitant and adjuvant temozolomide versus radiotherapy alone on survival in glioblastoma in a randomised phase III study: 5-year analysis of the EORTC-NCIC trial. Lancet Oncol. 10, 459-466 (2009).

2. Chinot, O. L. et al. Bevacizumab plus radiotherapy-temozolomide for newly diagnosed glioblastoma. N. Engl. J. Med. 370, 709-722 (2014).

3. Wen, P. Y. \& Kesari, S. Malignant gliomas in adults. N. Engl. J. Med. 359 492-507 (2008).

4. Aldape, K. et al. Challenges to curing primary brain tumours. Nat. Rev. Clin. Oncol. 16, 509-520 (2019)

5. Reardon, D. A. \& Wen, P. Y. Glioma in 2014: unravelling tumour heterogeneity-implications for therapy. Nat. Rev. Clin. Oncol. 12, 69-70 (2015).

6. Singh, S. K. et al. Identification of human brain tumour initiating cells. Nature 432, 396-401 (2004).

7. Bao, S. et al. Glioma stem cells promote radioresistance by preferential activation of the DNA damage response. Nature 444, 756-760 (2006).

8. Rich, J. N. Cancer stem cells in radiation resistance. Cancer Res. 67, 8980-8984 (2007).

9. Batlle, E. \& Clevers, H. Cancer stem cells revisited. Nat. Med. 23, 1124-1134 (2017).

10. Saygin, C., Matei, D., Majeti, R., Reizes, O. \& Lathia, J. D. Targeting cancer stemness in the clinic: from hype to hope. Cell Stem Cell 24, 25-40 (2019).

11. Prager, B. C., Xie, Q., Bao, S. \& Rich, J. N. Cancer stem cells: the architects of the tumor ecosystem. Cell Stem Cell 24, 41-53 (2019).

12. Circu, M. L. \& Aw, T. Y. Reactive oxygen species, cellular redox systems, and apoptosis. Free Radic. Biol. Med. 48, 749-762 (2010).

13. Schieber, M. \& Chandel, N. S. ROS function in redox signaling and oxidative stress. Curr. Biol. 24, R453-R462 (2014).

14. Finkel, T. Signal transduction by reactive oxygen species. J. Cell Biol. 194, 7-15 (2011).

15. Diehn, M. et al. Association of reactive oxygen species levels and radioresistance in cancer stem cells. Nature 458, 780-783 (2009).

16. Trachootham, D., Alexandre, J. \& Huang, P. Targeting cancer cells by ROSmediated mechanisms: a radical therapeutic approach? Nat. Rev. Drug Discov. 8, 579-591 (2009).

17. Sabharwal, S. S., Schumacker, P. T. \& Mitochondrial, R. O. S. in cancer: initiators, amplifiers or an Achilles' heel? Nat. Rev. Cancer 14, 709-721 (2014).

18. Galadari, S., Rahman, A., Pallichankandy, S. \& Thayyullathil, F. Reactive oxygen species and cancer paradox: to promote or to suppress? Free Radic. Biol. Med. 104, 144-164 (2017). 
19. Hayes, J. D., Dinkova-Kostova, A. T. \& Tew, K. D. Oxidative stress in cancer. Cancer Cell. 38, 167-197 (2020).

20. Immenschuh, S. \& Baumgart-Vogt, E. Peroxiredoxins, oxidative stress, and cell proliferation. Antioxid. Redox Signal. 7, 768-777 (2005).

21. Neumann, C. A. \& Fang, Q. Are peroxiredoxins tumor suppressors? Curr. Opin. Pharm. 7, 375-380 (2007).

22. Cox, A. G., Winterbourn, C. C. \& Hampton, M. B. Mitochondrial peroxiredoxin involvement in antioxidant defence and redox signalling. Biochem. J. 425, 313-325 (2009).

23. Whitaker, H. C. et al. Peroxiredoxin- 3 is overexpressed in prostate cancer and promotes cancer cell survival by protecting cells from oxidative stress. $\mathrm{Br} . J$. Cancer 109, 983-993 (2013).

24. Mishra, S., Murphy, L. C., Nyomba, B. L. \& Murphy, L. J. Prohibitin: a potential target for new therapeutics. Trends Mol. Med. 11, 192-197 (2005).

25. Artal-Sanz, M. \& Tavernarakis, N. Prohibitin and mitochondrial biology. Trends Endocrinol. Metab. 20, 394-401 (2009).

26. Theiss, A. L. \& Sitaraman, S. V. The role and therapeutic potential of prohibitin in disease. Biochim. Biophys. Acta 1813, 1137-1143 (2011)

27. Fusaro, G., Dasgupta, P., Rastogi, S., Joshi, B. \& Chellappan, S. Prohibitin induces the transcriptional activity of $\mathrm{p} 53$ and is exported from the nucleus upon apoptotic signaling. J. Biol. Chem. 278, 47853-47861 (2003).

28. Wang, S., Zhang, B. \& Faller, D. V. Prohibitin requires Brg-1 and Brm for the repression of E2F and cell growth. EMBO J. 21, 3019-3028 (2002).

29. Chiu, C. F. et al. Raf activation by Ras and promotion of cellular metastasis require phosphorylation of prohibitin in the raft domain of the plasma membrane. Oncogene 32, 777-787 (2013).

30. Yurugi, H. et al. Expression of prohibitins on the surface of activated $\mathrm{T}$ cells. Biochem. Biophys. Res. Commun. 420, 275-280 (2012).

31. Zhu, Z. et al. PHB associates with the HIRA complex to control an epigeneticmetabolic circuit in human ESCs. Cell Stem Cell 20, 274-289e277 (2017).

32. Zhan, X. et al. Glioma stem-like cells evade interferon suppression through MBD3/NuRD complex-mediated STAT1 downregulation. J. Exp. Med. 217, e20191340 (2020).

33. Man, J. et al. Hypoxic induction of vasorin regulates Notch1 turnover to maintain glioma stem-like cells. Cell Stem Cell 22, 104-118e106 (2018).

34. Zhou, W. et al. Targeting glioma stem cell-derived pericytes disrupts the blood-tumor barrier and improves chemotherapeutic efficacy. Cell Stem Cell 21, 591-603e594 (2017).

35. Wang, X. et al. Purine synthesis promotes maintenance of brain tumor initiating cells in glioma. Nat. Neurosci. 20, 661-673 (2017).

36. Jin, X. et al. Targeting glioma stem cells through combined BMI1 and EZH2 inhibition. Nat. Med. 23, 1352-1361 (2017).

37. Suva, M. L. et al. Reconstructing and reprogramming the tumor-propagating potential of glioblastoma stem-like cells. Cell 157, 580-594 (2014).

38. Chen, W. et al. Emerging role of microRNA-27a in human malignant glioma cell survival via targeting of prohibitin. Mol. Med. Rep. 12, 1515-1523 (2015).

39. Lathia, J. D., Mack, S. C., Mulkearns-Hubert, E. E., Valentim, C. L. \& Rich, J. N. Cancer stem cells in glioblastoma. Genes Dev. 29, 1203-1217 (2015).

40. Visvader, J. E. \& Lindeman, G. J. Cancer stem cells in solid tumours: accumulating evidence and unresolved questions. Nat. Rev. Cancer 8, 755-768 (2008).

41. Osman, C., Merkwirth, C. \& Langer, T. Prohibitins and the functional compartmentalization of mitochondrial membranes. J. cell Sci. 122, 3823-3830 (2009).

42. Kobayashi, C. I. \& Suda, T. Regulation of reactive oxygen species in stem cells and cancer stem cells. J. Cell. Physiol. 227, 421-430 (2012).

43. Takahashi, N. et al. Cancer cells co-opt the neuronal redox-sensing channel TRPA1 to promote oxidative-stress tolerance. Cancer Cell 33, 985-1003.e1007. (2018).

44. Schleicher, M. et al. Prohibitin-1 maintains the angiogenic capacity of endothelial cells by regulating mitochondrial function and senescence. J. Cell Biol. 180, 101-112 (2008).

45. Shadel, G. S. \& Horvath, T. L. Mitochondrial ROS signaling in organismal homeostasis. Cell 163, 560-569 (2015).

46. Nijtmans, L. G. et al. Prohibitins act as a membrane-bound chaperone for the stabilization of mitochondrial proteins. EMBO J. 19, 2444-2451 (2000).

47. Renschler, M. F. The emerging role of reactive oxygen species in cancer therapy. Eur. J. Cancer 40, 1934-1940 (2004).

48. Polier, G. et al. The natural anticancer compounds rocaglamides inhibit the Raf-MEK-ERK pathway by targeting prohibitin 1 and 2. Chem. Biol. 19, 1093-1104 (2012).

49. Thuaud, F., Ribeiro, N., Nebigil, C. G. \& Desaubry, L. Prohibitin ligands in cell death and survival: mode of action and therapeutic potential. Chem. Biol. 20, 316-331 (2013).
50. Iwasaki, S., Floor, S. N. \& Ingolia, N. T. Rocaglates convert DEAD-box protein eIF4A into a sequence-selective translational repressor. Nature 534, 558-561 (2016).

51. Baumann, B. et al. Rocaglamide derivatives are potent inhibitors of NF-kappa B activation in T-cells. The. J. Biol. Chem. 277, 44791-44800 (2002).

52. Wolfe, A. L. et al. RNA G-quadruplexes cause eIF4A-dependent oncogene translation in cancer. Nature 513, 65-70 (2014).

53. Moncunill-Massaguer, C. et al. A novel prohibitin-binding compound induces the mitochondrial apoptotic pathway through NOXA and BIM upregulation. Oncotarget 6, 41750-41765 (2015).

54. Bigarella, C. L., Liang, R. \& Ghaffari, S. Stem cells and the impact of ROS signaling. Development 141, 4206-4218 (2014).

55. Theiss, A. L. et al. Prohibitin protects against oxidative stress in intestinal epithelial cells. FASEB J. 21, 197-206 (2007).

56. Merkwirth, C. et al. Prohibitins control cell proliferation and apoptosis by regulating OPA1-dependent cristae morphogenesis in mitochondria. Genes Dev. 22, 476-488 (2008).

57. Pan, L., Woodard, J. L., Lucas, D. M., Fuchs, J. R. \& Kinghorn, A. D. Rocaglamide, silvestrol and structurally related bioactive compounds from Aglaia species. Nat. Prod. Rep. 31, 924-939 (2014).

\section{Acknowledgements}

We thank Dr. Jeremy Rich (University of California, San Diego) and Jennifer Yu (Cleveland Clinic) for kindly providing GSCs. We thank Dr. Shideng Bao (Cleveland Clinic) for kindly providing GSCs and NPCs, the helpful discussion and critical reading of the manuscript. We appreciate Dr. Xiuwu Bian (Army Medical University, Chongqing, China) for the GBM PDX. This research was supported by grants from the National Key Research and Development Program of China (2017YFA0505602), and National Natural Science Foundation of China (nos. 81872153, 81872408, and 81521064).

\section{Author contributions}

J.H.M., Q.X., and X.M.Z. supervised the project and acquired funding for this study. J.H.M., Q.X., and H.H.H. designed the experiments, analyzed the data and prepared the manuscript with contributions from all authors. H.H.H., S.Y.Z., Y.Y.L., Z.D.L., L.J.M., Y.C., X.Z.W., L.S.C. H.W.R., D.K.X., and Q.Y.H. performed the experiments. J.H.M., J.Q.W., and T.T.L. performed database analyses. F.Y.L. prepared GBM specimens. L.C., X.P., H.Y.L., T.L., K.H., A.L.L., X.M.Z., and T.Z. provided scientific advice for the manuscript. All authors read and approved the final manuscript.

\section{Competing interests}

The authors declare no competing interests.

\section{Additional information}

Supplementary information The online version contains supplementary material available at https://doi.org/10.1038/s41467-021-24108-6.

Correspondence and requests for materials should be addressed to Q.X. or J.M.

Peer review information Nature Communications thanks the anonymous reviewer(s) for their contribution to the peer review of this work.

Reprints and permission information is available at http://www.nature.com/reprints

Publisher's note Springer Nature remains neutral with regard to jurisdictional claims in published maps and institutional affiliations.

Open Access This article is licensed under a Creative Commons Attribution 4.0 International License, which permits use, sharing, adaptation, distribution and reproduction in any medium or format, as long as you give appropriate credit to the original author(s) and the source, provide a link to the Creative Commons license, and indicate if changes were made. The images or other third party material in this article are included in the article's Creative Commons license, unless indicated otherwise in a credit line to the material. If material is not included in the article's Creative Commons license and your intended use is not permitted by statutory regulation or exceeds the permitted use, you will need to obtain permission directly from the copyright holder. To view a copy of this license, visit http://creativecommons.org/ licenses/by/4.0/.

(C) The Author(s) 2021 